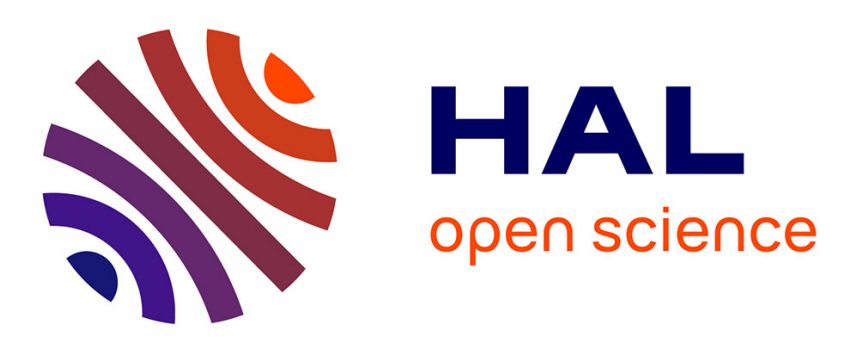

\title{
On the longitudinal optimal perturbations to inviscid plane shear flow: formal solution and asymptotic approximation
}

\author{
Cristobal Arratia, Jean-Marc Chomaz
}

\section{To cite this version:}

Cristobal Arratia, Jean-Marc Chomaz. On the longitudinal optimal perturbations to inviscid plane shear flow: formal solution and asymptotic approximation. Journal of Fluid Mechanics, 2013, 737, pp.387-411. 10.1017/jfm.2013.570 . hal-00994466

\section{HAL Id: hal-00994466}

https://hal-polytechnique.archives-ouvertes.fr/hal-00994466

Submitted on 30 May 2014

HAL is a multi-disciplinary open access archive for the deposit and dissemination of scientific research documents, whether they are published or not. The documents may come from teaching and research institutions in France or abroad, or from public or private research centers.
L'archive ouverte pluridisciplinaire $\mathbf{H A L}$, est destinée au dépôt et à la diffusion de documents scientifiques de niveau recherche, publiés ou non, émanant des établissements d'enseignement et de recherche français ou étrangers, des laboratoires publics ou privés. 


\title{
On the longitudinal optimal perturbations to inviscid plane shear flow: formal solution and asymptotic approximation
}

\author{
C. Arratia ${ }^{1,2, \dagger}$ and J.-M. Chomaz ${ }^{1}$ \\ ${ }^{1}$ Laboratoire d'Hydrodynamique (LadHyX), École Polytechnique-CNRS, F-91128 Palaiseau, France \\ ${ }^{2}$ LFMI, École Polytechnique Fédérale de Lausanne, CH1015 Lausanne, Switzerland
}

(Received 13 December 2012; revised 7 September 2013; accepted 23 October 2013; first published online 26 November 2013)

We study the longitudinal linear optimal perturbations (which maximize the energy gain up to a prescribed time $T$ ) to inviscid parallel shear flow, which present unbounded energy growth due to the lift-up mechanism. Using the phase invariance with respect to time, we show that for an arbitrary base flow profile and optimization time, the computation of the optimal longitudinal perturbation reduces to the resolution of a single one-dimensional eigenvalue problem valid for all times. The optimal perturbation and its amplification are then derived from the lowest eigenvalue and its associated eigenfunction, while the remainder of the infinite set of eigenfunctions provides an orthogonal base for decomposing the evolution of arbitrary perturbations. With this new formulation we obtain, asymptotically for large spanwise wavenumber $k_{z}$, a prediction of the optimal gain and the localization of inviscid optimal perturbations for the two main classes of parallel flows: free shear flow with an inflectional velocity profile, and wall-bounded flow with maximum shear at the wall. We show that the inviscid optimal perturbations are localized around the point of maximum shear in a region with a width scaling like $k_{z}^{-1 / 2}$ for free shear flow, and like $k_{z}^{-2 / 3}$ for wall-bounded shear flows. This new derivation uses the stationarity of the base flow to transform the optimization of initial conditions in phase space into the optimization of a temporal phase along each trajectory, and an optimization among all trajectories labelled by their intersection with a codimension-1 subspace. The optimization of the time phase directly imposes that the initial and final energy growth rates of the optimal perturbation should be equal. This result requires only time invariance of the base flow, and is therefore valid for any linear optimal perturbation problem with stationary base flow.

Key words: boundary layers, instability, shear layers

\section{Introduction}

Many studies have demonstrated that linear perturbations to parallel shear flow can exhibit very large energy growth in the absence of unstable eigenmodes (Gustavsson 1991; Farrell \& Ioannou 1993; Reddy \& Henningson 1993; Schmid \& Henningson 2001). The mechanism responsible for the largest energy growth is essentially the 
same for all inviscid or viscous shear flows at sufficiently large Reynolds number $R e$ : the forcing of cross-stream (or wall-normal) vorticity associated with low and high speed streamwise streaks by cross-stream velocity varying in the span. This so-called lift-up mechanism is more efficient for perturbations elongated in the streamwise direction. It can also be understood as the flow induced by streamwise vorticity that, superposed on positive shear, lifts up fluid at low speed while pushing down high-velocity fluid.

An early description of what is now known as the lift-up mechanism was given in the context of the rapid distortion of turbulence by Moffatt (1967), who described the evolution of single Fourier components under the effect of constant shear, and showed that for the Fourier components with streamwise wavenumber $k_{x}=0$, the inviscid linear energy growth is unbounded, concentrated in the streamwise velocity and mediated by (streamwise) vortices parallel to the mean flow. In the context of the stability of plane parallel flow, Ellingsen \& Palm (1975) showed that for inviscid perturbations which are independent of the streamwise coordinate, the streamwise velocity grows linearly in time; they concluded that the base flow is unstable to such perturbations even if it has no inflection point. Ellingsen \& Palm (1975) provided an example of a finite amplitude solution, and remarked that the total (base flow plus perturbation) streamwise velocity of material particles is conserved. Still in the inviscid case, Landahl (1980) showed that the integrals along the streamwise direction of localized perturbations satisfy the same equations as the streamwise independent perturbations (i.e. perturbations infinitely elongated in the streamwise direction). He then showed that the constant growth of the perturbation integral corresponds to a streamwise spreading of the disturbance, and that the integrated energy grows faster than linearly in time.

Most subsequent work deals with viscous flows. For wall-bounded parallel flows, it has been shown that for large $R e$ the largest possible amplification due to lift-up scales as $R e^{2}$, and is attained after a time scaling as $\sim R e$ (Gustavsson 1991; Reddy $\&$ Henningson 1993). The dynamics of these perturbations consists of an initial phase of energy growth due to the inviscid lift-up mechanism and a later decay due to viscous diffusion. For plane Couette and Poiseuille flow, the spanwise wavenumber $k_{z}$ corresponding to this largest possible amplification is of order one in units of the distance between walls (Reddy \& Henningson 1993). The situation changes if instead of focusing on the largest transient growth for all times, one looks for the most amplified perturbation up to a prescribed optimization time $T$. In that case the most amplified $k_{z}$ is a function of $T$, and it results from the competition between the inviscid lift-up mechanism tending to select small-scale structures efficiently localized around the maximum shear, and viscous effects damping small-scale structures. The same competition will determine the $k_{z}$ of the maximum instantaneous growth rate, which corresponds to the optimal perturbation in the limit $T \rightarrow 0$.

The initial value problem in the case of inviscid longitudinal perturbations to an arbitrary parallel shear flow admits very simple solutions in closed form (Ellingsen \& Palm 1975; Schmid \& Henningson 2001). Concerning the optimal perturbation problem, the analytical expression for the optimal inviscid energy growth for any optimization time $T$ has been given only in the case of unbounded uniform shear flow by Farrell \& Ioannou (1993). Their formula has no dependence on the spanwise wavenumber because there is a time scale but no length scale associated with such a uniform shear flow. Other works dealing with the inviscid limit of optimal perturbations of the lift-up type in compressible flow have relied on numerical computations even for the inviscid limit case, as done for example by Hanifi \& 
Henningson (1998) for boundary layers and by Malik, Alam \& Dey (2006) for nonisothermal plane Couette flow. Still, to the authors' knowledge, the problem of the optimal perturbations in the case of inviscid incompressible flow with an arbitrary profile has not been further analysed in the literature.

Here we solve the problem of the optimal longitudinal (streamwise independent) perturbations for an arbitrary inviscid shear flow. In $\S 2$, we recall first the solution for inviscid longitudinal perturbations to plane parallel flow. Then, using the time invariance of the governing equations, we derive a generic property of the optimal linear perturbations to any stationary base flow. We use this property to reformulate the optimization problem in the whole phase space into an optimization in a codimension-1 subspace and in an initial time phase along trajectories passing through points in that subspace. The reformulated optimization problem leads to a differential eigenvalue problem in one dimension whose solutions provide an orthogonal basis to describe the evolution of the optimal and sub-optimal perturbations. In $\S 3$ we consider plane Couette flow, a specific shear layer profile (introduced for its exact solution), and plane Poiseuille flow as examples, and we provide the exact solutions allowing the construction of the inviscid optimal perturbation for any optimization time. In $\S 4$ we consider arbitrary plane parallel shear flow by performing a local approximation around the maximum shear, which may be localized either at a wall (wall-bounded shear flow) or far away from it (free shear flow). In both cases we provide asymptotic solutions for the eigenmode for large $k_{z}$, giving estimates of the optimal amplification and of the localization width of the optimal perturbation around the maximum shear. The asymptotic predictions for free and wall-bounded shear flows agree, for large $k_{z}$, with the optimal perturbations to a tanh profile solved directly by the direct-adjoint technique implemented on a three-dimensional pseudospectral code, and with the exact optimal perturbations to Poiseuille flow given in $\S 3$. The effect of viscosity is considered in $\S 5$, where we show that the optimal gain for a given optimization time $T$ may be derived from the inviscid case and that the viscous optimal perturbations approach our inviscid prediction as $\operatorname{Re} \rightarrow \infty$ for free shear flow, whereas for wall-bounded flow the optimal perturbation differs only by the occurrence of a boundary layer next to a wall required to satisfy the no-slip boundary conditions. Finally in $\S 6$ we draw our conclusions.

\section{Formulation}

We consider infinitesimal perturbations $\boldsymbol{u}=\operatorname{Re}\left\{[u, v, w] \exp \left(\mathrm{i} k_{x} x+\mathrm{i} k_{z} z\right)\right\}$ to parallel inviscid flow $\boldsymbol{U}=U(y) \boldsymbol{e}_{x}$ with shear $S(y)=U^{\prime}$ (where $(\cdot)^{\prime}$ denotes the $y$-derivative). In the case of longitudinal perturbations $k_{x}=0$, the linearized Euler equations classically reduce to (Schmid \& Henningson 2001)

$$
\begin{aligned}
\partial_{t} u(y, t) & =-S(y) v(y, t), \\
\partial_{t} v(y, t) & =0, \\
\partial_{t} w(y, t) & =0, \\
\partial_{y} v(y, t)+\mathrm{i} k_{z} w(y, t) & =0,
\end{aligned}
$$

where the domain is $y \in\left(y_{1}, y_{2}\right)$ and infinite in $x$ and $z$. Free-slip boundary conditions are imposed so that

$$
v\left(y_{1}, t\right)=v\left(y_{2}, t\right)=0
$$


The general solution of $(2.1)$ is

$$
\begin{aligned}
u(y, t) & =u_{o}(y)-S(y) v_{o}(y) t, \\
v(y, t) & =v_{o}(y), \\
w(y, t) & =\mathrm{i} v_{o}^{\prime}(y) / k_{z},
\end{aligned}
$$

where $u_{o}(y)$ and $v_{o}(y)$ denote, respectively, the streamwise and cross-stream velocities at $t=0$. The entire phase space $\mathscr{P}$ can be reduced to the $(u, v)$-space since $w$ is given by $v$, which in turn stays constant giving trajectories embedded in the subspace wherein $v=v_{o}$. Thus, once $v_{o}$ is given, this solution may be represented by a trajectory in the $u$-space only.

The energy $E$ of solution (2.3) is

$$
E(t)=E_{o}-\operatorname{Re}\left(\left\langle u_{o}, S v_{o}\right\rangle\right) t+\frac{\left\|S v_{o}\right\|^{2}}{2} t^{2}
$$

where $2 E_{o}=\left\|u_{o}\right\|^{2}+\left\|v_{o}\right\|^{2}+\left\|v_{o}^{\prime}\right\|^{2} / k_{z}^{2},\|\cdot\|$ being the norm $\|f\|^{2}=\langle f, f\rangle$ associated with the inner product $\langle f, g\rangle$ between any two functions $f$ and $g$ defined as

$$
\langle f, g\rangle=\int_{y_{1}}^{y_{2}} f(y)^{*} g(y) \mathrm{d} y,
$$

where $(\cdot)^{*}$ denotes complex conjugation. From now on we will assume $\left\|S v_{o}\right\| \neq 0$ since this case is degenerate with $S v_{o}=0$ everywhere, solution (2.3) stationary and constant energy $E_{o}$, see (2.4).

We can define a shifted time variable $\bar{t} \equiv t+\bar{t}_{o}$, where

$$
\bar{t}_{o}=-\frac{\operatorname{Re}\left(\left\langle u_{o}, S v_{o}\right\rangle\right)}{\left\|S v_{o}\right\|^{2}},
$$

such that the energy along the trajectory passing through the initial condition $\boldsymbol{u}_{o}=\left(u_{o}, v_{o}, \mathrm{i} v_{o}^{\prime} / k_{z}\right)$ is

$$
E(t)=\bar{E}(\bar{t})=\bar{E}_{o}+\frac{\left\|S v_{o}\right\|^{2}}{2} \bar{t}^{2},
$$

which is minimized at $\bar{t}=0$ with a minimum $\bar{E}_{o}$ given by

$$
2 \bar{E}_{o}=\left\|\bar{u}_{o}\right\|^{2}+\left\|v_{o}\right\|^{2}+\frac{\left\|v_{o}^{\prime}\right\|^{2}}{k_{z}^{2}},
$$

where

$$
\bar{u}_{o} \equiv u_{o}-S v_{o} \frac{\operatorname{Re}\left(\left\langle u_{o}, S v_{o}\right\rangle\right)}{\left\|S v_{o}\right\|^{2}}=u_{o}+S v_{o} \bar{t}_{o}
$$

is the streamwise velocity at the new time origin $\bar{t}=0$, which corresponds to $t=-\bar{t}_{o}$ and may be negative or positive, i.e. in the past or in the future relative to the initial condition, now at $\bar{t}=\bar{t}_{o}$ in the shifted time variable. We stress that $\bar{E}_{o} \leqslant E(t)$ for all $t$. Accordingly, the streamwise kinetic energy reaches its minimum $\left\|\bar{u}_{o}\right\|^{2} / 2$ at $\bar{t}=0$ (equivalently at $t=-\bar{t}_{o}$ ). It can also be noted that $\operatorname{Re}\left\{\left\langle\bar{u}_{o}, S v_{o}\right\rangle\right\}=0$, meaning that at $\bar{t}=0$ the streamwise velocity $u=\bar{u}_{o}$ is orthogonal to its time-varying part $S v_{o} t$ (equation $(2.3 a)$ ) with respect to the inner product $\int\left[f_{r} g_{r}+f_{i} g_{i}\right] \mathrm{d} y=\operatorname{Re}\{\langle f, g\rangle\}$, which corresponds to the two-dimensional Euclidean inner product considering the complex functions $f(y)$ and $g(y)$ as real vector fields of two components of the form $f=\left(f_{r}, f_{i}\right)$ 


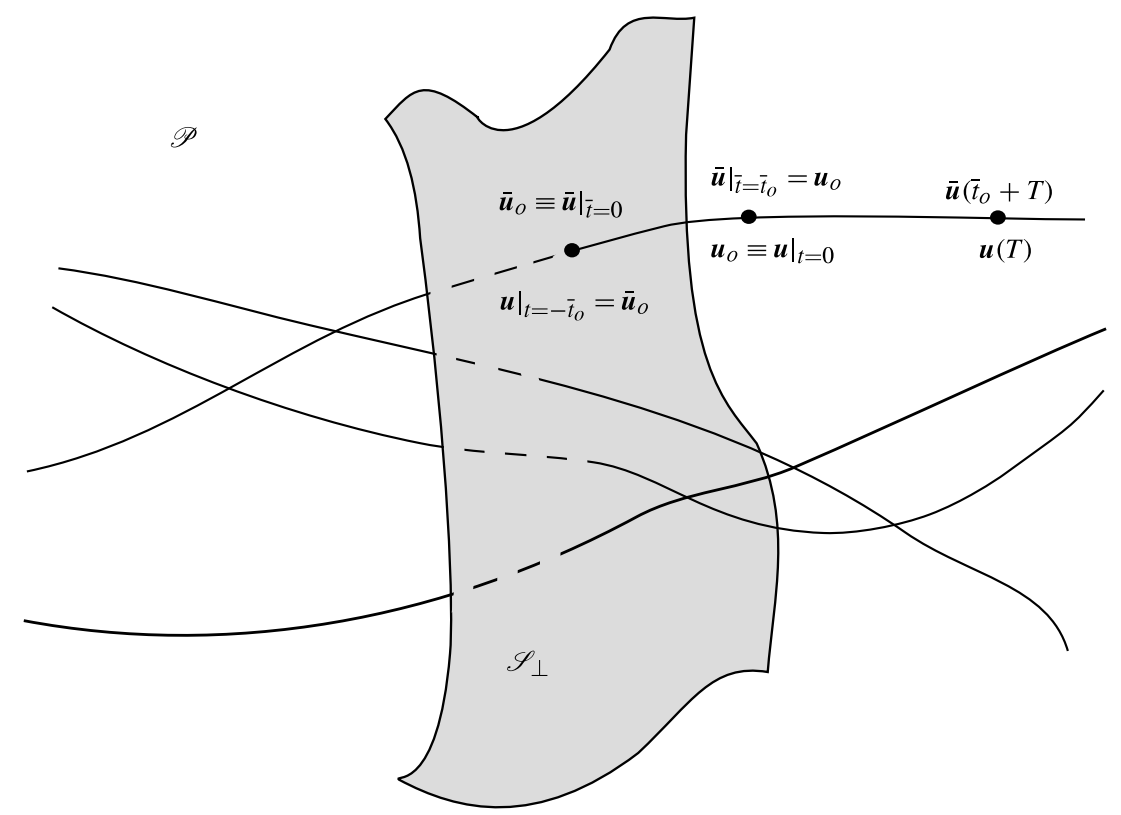

FIGURE 1. Schematic representation of different trajectories in phase space $\mathscr{P}$ and the codimension-1 subspace $\mathscr{S}_{\perp}$ of the $\overline{\boldsymbol{u}}_{o}$ such that $\operatorname{Re}\left\{\left\langle\bar{u}_{o}, S v_{o}\right\rangle\right\}=0$, i.e. such that $\bar{u}_{o}$ and $S v_{o}$ are orthogonal in the two-dimensional Euclidean inner product. The trajectory passing through the initial condition $\boldsymbol{u}_{o}$ in (2.10) intersects $\mathscr{S}_{\perp}$ at $t=-\bar{t}_{o}$, at the point $\overline{\boldsymbol{u}}_{o}$ of (2.11).

with $f_{r}=\operatorname{Re}(f)$ and $f_{i}=\operatorname{Im}(f)$. To distinguish the two inner products we will hereafter call $\langle f, g\rangle$ the complex inner product and $\operatorname{Re}\{\langle f, g\rangle\}$ the two-dimensional Euclidean inner product.

\subsection{Reformulating the optimization problem}

Consider the problem of computing the optimal gain at a finite time $T$ :

$$
G_{o p t}(T)=\max _{\boldsymbol{u}_{o} \in \mathscr{P}}\left(\frac{E(T)}{E(0)}\right),
$$

where $\mathscr{P}$ represents the set of all perturbations $\boldsymbol{u}_{o}$ satisfying the incompressibility condition $(2.1 d)$ and is therefore, as already stated, determined by the two-component vector field $(u, v)$ varying in $y$ and fulfilling the boundary condition (2.2). The optimization problem (2.10) is equivalent to

$$
G_{o p t}(T)=\max _{\bar{t}_{o} \in \mathbb{R}, \bar{u}_{o} \in \mathscr{S}_{\perp}}\left(\frac{\bar{E}\left(T+\bar{t}_{o}\right)}{\bar{E}\left(\bar{t}_{o}\right)}\right),
$$

meaning that any element of $\mathscr{P}$ can be defined by a starting time $\bar{t}_{o}$ and a shifted condition at $\bar{t}=0, \overline{\boldsymbol{u}}_{o}=\left(\bar{u}_{o}, v_{o}, \mathrm{i} v_{o}^{\prime} / k_{z}\right)$, in the codimension-1 subspace $\mathscr{S}_{\perp}$ such that $\operatorname{Re}\left(\left\langle\bar{u}_{o}, S v_{o}\right\rangle\right)=0$, i.e. such that $\bar{u}_{o}$ and $S v_{o}$ are orthogonal in the two-dimensional Euclidean inner product. As illustrated in figure 1, since the base flow is stationary, any initial condition $\boldsymbol{u}_{o}$ in (2.10) belongs to a unique trajectory passing through a point $\overline{\boldsymbol{u}}_{o}$ in $\mathscr{S}_{\perp}$ at the time $t=-\bar{t}_{o}$ (or $\bar{t}=0$ ). Equation (2.11) is a decomposition of the optimization problem in the whole phase space into the optimization among trajectories defined by their intersection with the codimension- 1 subspace $\mathscr{S}_{\perp}$ and a 
time phase $\bar{t}_{o}$ defining the position of the initial condition along the trajectory. This procedure is valid for systems with time-invariant evolution equations (a condition which corresponds here to the requirement of a steady base flow). The subspace $\mathscr{S}_{\perp}$ can then be thought of as a Poincaré section allowing the labelling of trajectories by their crossing of the section, although in this case each trajectory crosses the section only once so the concept of the return map does not apply here.

We shall now maximize $\bar{E}\left(T+\bar{t}_{o}\right) / \bar{E}\left(\bar{t}_{o}\right)$. Note first that, for $\bar{t}_{o p t}$ achieving the maximum (2.11), the derivative with respect to $\bar{t}_{o}$ should vanish, i.e.

$$
\frac{\partial}{\partial \bar{t}_{o}} \frac{\bar{E}\left(T+\bar{t}_{o}\right)}{\bar{E}\left(\bar{t}_{o}\right)}=0
$$

implying that the instantaneous growth rate of the perturbation,

$$
\sigma(t)=\frac{1}{2 E(t)} \frac{\partial E(t)}{\partial t}
$$

should be equal at the initial and final times, i.e.

$$
\sigma\left(\bar{t}_{\text {opt }}\right)=\sigma\left(T+\bar{t}_{\text {opt }}\right) .
$$

Condition (2.14) is only a necessary condition. It generalizes to any linear optimization problem (2.10) with time-independent equations of motion, since at the maximum of $\bar{E}\left(T+\bar{t}_{o}\right) / \bar{E}\left(\bar{t}_{o}\right)$ along a single trajectory, the gain should not vary when the starting point along the trajectory is infinitesimally varied. For a stationary flow, linear optimal perturbations at any finite optimization time $T$ should be such that the initial and final instantaneous growth rates are equal, which may seem somewhat counter-intuitive. On any trajectory, (2.14) will in general be fulfilled only on a discrete set of points $\bar{t}_{o}=\bar{t}_{o \sigma}^{n}$, allowing for the optimization along the trajectories to be solved first. In nonlinear cases, (2.14) is still valid if the optimal gain is to be determined without imposing the energy of the initial perturbation, but in practice this initial energy is often fixed and the final energy maximized. In that case, the fixed energy of the initial condition will constrain the available starting points along trajectories, and (2.14) will not be in general satisfied.

In the present simple case, the necessary condition (2.14) indeed allows us to solve for possible $\bar{t}_{o}$ before solving for $\overline{\boldsymbol{u}}_{o}$. For any element of $\mathscr{S}_{\perp},(2.14)$ is attained for two particular values of $\bar{t}_{o}$ :

$$
\bar{t}_{o}=\bar{t}_{\sigma \sigma}^{ \pm} \equiv-\frac{T}{2} \pm \sqrt{\left(\frac{T}{2}\right)^{2}+\tau^{2}},
$$

where $\tau=\sqrt{2 \bar{E}_{o} /\left\|S v_{o}\right\|^{2}}$ is the time at which the instantaneous growth rate $\sigma(\bar{t}=\tau)=1 / 2 \tau$ is maximum along each trajectory. The optimal initial condition for a given $\overline{\boldsymbol{u}}_{o} \in \mathscr{S}_{\perp}$ is given by the upper + sign, the - sign corresponding to an evolution interval $\left[\bar{t}_{o \sigma}, \bar{t}_{o \sigma}+T\right]$ in the negative $\bar{t}$ domain in which $\bar{E}(\bar{t})$ decays according to (2.7). The - sign solution $\bar{t}_{o \sigma}^{-}$satisfies the necessary condition (2.14) but corresponds to the minimum of the gain along each trajectory, not to the maximum.

Replacing $\bar{t}_{o} \rightarrow \bar{t}_{o \sigma}^{+}$in the optimization problem (2.11) yields

$$
G_{o p t}(T)=\max _{\bar{u}_{o} \in \mathscr{S}_{\perp}}\left(\bar{G}_{o \sigma}\right),
$$


where

$$
\bar{G}_{o \sigma} \equiv \frac{\bar{E}\left(\bar{t}_{o \sigma}^{+}+T\right)}{\bar{E}\left(\bar{t}_{o \sigma}^{+}\right)}=1+\frac{T^{2}}{2 \tau^{2}}+\frac{T}{\tau} \sqrt{1+\left(\frac{T}{2 \tau}\right)^{2}} .
$$

Equations (2.16) show that all the degrees of freedom, i.e. the $\overline{\boldsymbol{u}}_{o} \in \mathscr{S}_{\perp}$, enter the optimization problem through a single parameter $\tau$. For a given optimization time $T$, the gain $\bar{G}_{o \sigma}$ is a decreasing function of $\tau$ and the optimization problem therefore reduces to finding $\overline{\boldsymbol{u}}_{o} \in \mathscr{S}_{\perp}$ which minimizes $\tau$. Note also that minimizing $\tau$ will single out the trajectory in which the maximum growth rate over the entire phase space $\sigma_{\max }=\max (\sigma)$ occurs. In terms of the components of $\overline{\boldsymbol{u}}_{o}$, we have

$$
\tau^{2}=\frac{\left\|\bar{u}_{o}\right\|^{2}+\left\|v_{o}\right\|^{2}+k_{z}^{-2}\left\|v_{o}^{\prime}\right\|^{2}}{\left\|S v_{o}\right\|^{2}},
$$

requiring that $\bar{u}_{o}=0$ for the optimal perturbations (minimizing $\tau$ ). Therefore, for any $v_{o}$ the optimization over $\bar{u}_{o}$ gives $\bar{u}_{o}=0$ and the optimal perturbation problem is now reduced to the variational problem of finding the $v_{o}$ that minimizes

$$
\tau^{2}=\tau_{o}^{2} \equiv \frac{\left\|v_{o}\right\|^{2}+k_{z}^{-2}\left\|v_{o}^{\prime}\right\|^{2}}{\left\|S v_{o}\right\|^{2}} .
$$

This is a standard variational problem, which can be formulated by writing the functional $\tau_{o}^{2}$ in terms of $v_{o}=v_{o p t}+\delta v$, where $v_{o p t}$ is the optimal $v_{o}$ yielding $\tau_{o p t}=\min \left(\tau_{o}\right)$, and $\delta v$ is an arbitrary variation. The first (i.e. $O(\delta v)$ ) term in the expansion of $\tau_{o}^{2}$ about $v_{\text {opt }}$ gives the functional derivative $\delta \tau_{o}^{2} / \delta v$ evaluated at $v_{\text {opt }}$. The optimality condition requires this functional derivative to be zero, i.e. $\delta \tau_{o}^{2} /\left.\delta v\right|_{v_{\text {opt }}}=0$ for all $\delta v$. After integration by parts and application of the boundary conditions (2.2) to $\delta v$, the optimality condition is equivalent to an eigenvalue problem for $\tau_{o p t}^{2}$ where the eigenfunction is $v_{\text {opt }}$,

$$
v_{o p t}^{\prime \prime}+k_{z}^{2}\left(\tau_{o p t}^{2} S(y)^{2}-1\right) v_{o p t}=0
$$

with boundary conditions (2.2).

Equation (2.19) is a key result of the paper that will allow us to find new exact solutions and predict scaling laws for the optimal perturbations. It is valid for the longitudinal optimal perturbations to any shear profile $S(y)$ and for any optimization time $T$. Therefore, the wall-normal optimal velocity $v_{o p t}$ is independent of $T$ and so is the $w$ component given by $w_{\text {opt }}=\mathrm{i} v_{o p t}^{\prime} / k_{z}$. Accordingly, the optimal perturbations for different optimization time $T$ all belong to the same trajectory in phase space. The only dependence on $T$ is through the phase shift $\bar{t}_{\text {opt }}$ given directly by (2.15) with $\tau=\tau_{\text {opt }}$ as

$$
\bar{t}_{o p t}=-\frac{T}{2}+\sqrt{\left(\frac{T}{2}\right)^{2}+\tau_{o p t}^{2}},
$$

which induces a change only in the $u$ component of the optimal perturbation

$$
u_{\text {opt }}(y, t)=-S(y) v_{\text {opt }}(y)\left(t+\bar{t}_{\text {opt }}\right) \text {. }
$$

The optimal gain for optimization time $T$ is then given by (2.16b) as

$$
G_{o p t}(T)=1+\frac{T^{2}}{2 \tau_{o p t}^{2}}+\frac{T}{\tau_{o p t}} \sqrt{1+\left(\frac{T}{2 \tau_{o p t}}\right)^{2}} .
$$




\subsection{About the optimal perturbation equation (2.19)}

Equation (2.19) is a generalized eigenvalue problem of the Sturm-Liouville type (Teschl 2012, §5), with the time scale $\tau_{\text {opt }}$ as the eigenvalue and the optimal perturbation $v_{\text {opt }}(y)$ as the eigenfunction. Since the equation coefficients and the independent variable $y$ are real, we can consider real solutions without loss of generality. In general, there is an infinite countable set of eigenvalues $\tau_{n}$ with its corresponding set of eigenfunctions $\left\{v_{n}\right\}$ forming an orthogonal basis of functions localized where $S(y) \neq 0$. In appendix A we show how the $\left\{v_{n}\right\}$ basis can be used to construct an orthogonal decomposition of the evolution of general perturbations.

A physical interpretation of (2.19) is given by analogy to the Schrödinger equation governing the energy eigenstates of a quantum particle of mass $m$. Indeed, the optimal perturbation $v_{\text {opt }}$ corresponds to the ground state wavefunction of a particle with energy -1 in a negative potential $-\tau_{o p t}^{2} S(y)^{2}$. Because the energy of the particle must be larger than the minimum of the potential, we have

$$
\tau_{\text {opt }}^{2}>\frac{1}{S_{\max }^{2}},
$$

where $S_{\max }^{2}$ is the maximum of $S^{2}$. In the quantum particle analogy, $k_{z}^{2}$ is replaced by $2 m / \hbar^{2}$, which indicates that increasing $k_{z}$ corresponds to decreasing $\hbar$ or increasing the mass. This suggests that increasing $k_{z}$ allows for a more 'concentrated' $v_{\text {opt }}$ (in the sense of a more localized eigenfunction) around the maximum of the shear (the minimum of the potential $-\tau_{o p t}^{2} S^{2}$ ), in agreement with what one may expect for the lift-up mechanism. It is interesting to note that the $n$th eigenfunction of (2.19) corresponds to a quantum bound state of a particle whose energy is always -1 , but in the $n$-dependent potential well $-\tau_{n}^{2} S^{2}$, whose depth increases with $n$. This remark is relevant for the case in which $S(y)=0$ in part of the domain, and explains why in the present case the discrete and infinite eigenmodes are always localized in the region with shear (inside the potential well), whereas in an analogous quantum potential there would only be a finite number of localized bound states (with negative energy). A specific example of this is shown in $\$ 3.2$ below.

\section{Base flow examples: Couette, free shear layer and Poiseuille flow}

\subsection{Couette}

We consider plane Couette flow in the region $y \in[0,1]$. For plane Couette flow the shear rate $S$ is constant and (2.19) has infinitely many solutions of the form

$$
\begin{gathered}
v_{n}=\sin (n \pi y), \\
\tau_{n}^{2}=\frac{1}{S^{2}}\left(1+\frac{n^{2} \pi^{2}}{k_{z}^{2}}\right)
\end{gathered}
$$

for $n=1,2, \ldots, \infty$. The optimal cross-stream velocity $v_{\text {opt }}=v_{1}$ is then obtained by evaluating (3.1a) at $n=1$. Similarly, the eigenvalue leading to the optimal amplification is given by

$$
\tau_{\text {opt }}=\tau_{1}=\frac{1}{|S|} \sqrt{1+\left(\frac{\pi}{k_{z}}\right)^{2}}
$$


which, after substitution in (2.22), yields the optimal gain at time $T$

$$
G_{\text {opt }}(T)=1+\frac{T^{2} S^{2}+T|S| \sqrt{T^{2} S^{2}+4\left(1+\lambda^{2} / 4\right)}}{2\left(1+\lambda^{2} / 4\right)},
$$

where $\lambda=2 \pi / k_{z}$ is the spanwise wavelength of the perturbation. From (2.21), the optimal initial streamwise velocity is given by

$$
\left.u_{\text {opt }}\right|_{t=0}=S v_{\text {opt }}\left[\frac{T}{2}-\left(\frac{1+\lambda^{2} / 4}{S^{2}}+\frac{T^{2}}{4}\right)^{1 / 2}\right] .
$$

Equation (3.4) is similar to the expression given by Farrell \& Ioannou (1993, equation (19)) for the optimal initial condition in the constant shear case. Their expression within square brackets reduces to ours in (3.4) if one requires that their crossstream wavenumber (which we would call $k_{y}$ ) satisfies the boundary conditions for $v$. However, they do not give explicitly the ratio between the amplitudes of the different components of the flow, so a full comparison with their solution can not be made directly. Still, the correspondence of the two square brackets confirms, up to a multiplicative constant, the dependence of $\left.u_{\text {opt }}\right|_{t=0}$ on the different parameters.

\subsection{A free shear layer example}

We consider an example of a free shear or mixing layer that gives rise to exact analytical solutions, a velocity profile given by $U(y)=2 U_{o} \arctan (\tanh (y / 2 L))$, which we will refer to as the arcttanh profile. This profile connects two semi-infinite regions at $|y| \gg 1$ with different constant velocities $U(y \rightarrow \pm \infty)= \pm \pi U_{o} / 2$. The shear associated to the arcttanh velocity profile is

$$
S(y) \equiv U^{\prime}(y)=S_{o} \operatorname{sech}(y / L)
$$

where $S_{o}=U_{o} / L$ is the maximum shear. Equation (2.19) then becomes

$$
v^{\prime \prime}+k_{z}^{2}\left(\tau^{2} S_{o}^{2} \operatorname{sech}^{2}(y / L)-1\right) v=0
$$

with $v(y \rightarrow \pm \infty) \rightarrow 0$ as boundary conditions. A similar problem has been treated in the quantum mechanics textbook of Landau \& Lifshitz (1977, at the end of $\S 23$ ). Solutions satisfying the boundary conditions are given by

$$
v_{n}(y)=\left(1-\tanh ^{2}(y / L)\right)^{\epsilon / 2} F(-n+1, \epsilon+s+1 ; \epsilon+1 ;[1+\tanh (y / L)] / 2),
$$

where $\epsilon=\left|L k_{z}\right|, s=-1 / 2+\sqrt{L^{2} k_{z}^{2} S_{o}^{2} \tau^{2}+1 / 4}, n \geqslant 1$ is an integer and $F(a, b ; c ; \xi)$ is the hypergeometric function (in the notation of Abramowitz \& Stegun 1964, § 15), which in this case of $a=-n+1$ becomes a polynomial in $\xi$ of order $n-1$. Note that the prefactor $\left[1-\tanh ^{2}(y / L)\right]^{\epsilon / 2}$ localizes $v_{n}$ around the shear layer at $y=0$. The eigenvalues are determined by the condition

$$
\epsilon=s-n+1,
$$

where $\epsilon$ has to be positive. For the quantum mechanical case treated in Landau $\&$ Lifshitz (1977), the eigenvalues (energy levels) are determined through $\epsilon$ with $s$ fixed by the binding potential; therefore the condition (3.8) yields a finite number of solutions (bound states). In the present case, the eigenvalues $\tau_{n}$ are determined through $s$ with $\epsilon>0$ fixed; therefore, condition (3.8) yields an infinite set of eigenvalues which, 
after replacement, is given by

$$
\tau_{n}=\frac{1}{\left|S_{o}\right|} \sqrt{1+\frac{2 n-1}{\left|L k_{z}\right|}+\frac{n(n-1)}{\left(L k_{z}\right)^{2}}},
$$

where $n=1,2, \ldots, \infty$. Thus, as anticipated in $\S 2.2$, there is an infinite number of eigenfunctions which are all localized around the shear layer.

For the optimal perturbation the leading solution is for $n=1$, which gives

$$
\tau_{o p t}=\tau_{1}=\frac{1}{\left|S_{o}\right|} \sqrt{1+\frac{1}{\left|L k_{z}\right|}},
$$

and

$$
v_{\text {opt }}=v_{1}=\left(1-\tanh ^{2}(y / L)\right)^{\left|L k_{z}\right| / 2} .
$$

Given the right choice of $U_{o}$ and $L$ for the arcttanh profile, solution (3.10) provides an excellent approximation for $v_{o p t}$ and the optimal gain for a tanh profile, which is commonly used as a simple model of mixing layers. This is shown in appendix B.

\subsection{Plane Poiseuille flow}

We consider plane Poiseuille flow $U(y)=1-y^{2}$ for $y \in[-1,1]$. Equation (2.19) can be written as

$$
v^{\prime \prime}+\left(\bar{y}^{2}-\alpha\right) v=0,
$$

where $\bar{y}=\sqrt{2 \tau k_{z}} y, \alpha=k_{z} / 2 \tau$ and the boundary conditions are

$$
\left.v\right|_{\bar{y}= \pm \sqrt{2 \tau k_{z}}}=0 .
$$

The even solutions of $(3.11 a)$, corresponding to $\tau_{m}$ with $m$ odd and such that $v(\bar{y})=v(-\bar{y})$, are given by

$$
v(\bar{y})=\exp \left(-\mathrm{i} \frac{\bar{y}^{2}}{2}\right) M\left(-\mathrm{i}(\mathrm{i}+\alpha) / 4,1 / 2, \mathrm{i} \bar{y}^{2}\right),
$$

where $M(a, b, z)$ is the confluent hypergeometric function of the first kind (also called Kummer's function: see Abramowitz \& Stegun 1964, § 13). For a given $\alpha$, the corresponding $\tau_{2 n-1}$ and $k_{z}$ are obtained as

$$
\begin{aligned}
\tau_{2 n-1} & =\frac{\iota_{n}}{2 \sqrt{\alpha}}, \\
k_{z} & =\iota_{n} \sqrt{\alpha},
\end{aligned}
$$

where $\iota_{n}$ is the $n$th zero of $M\left(-\mathrm{i}(\mathrm{i}+\alpha) / 4,1 / 2, \mathrm{i} \bar{y}^{2}\right)$. The computation of the first zero $\iota_{1}$ and the evaluation of (3.12) were performed with the commercial software Mathematica, allowing us to obtain the optimal perturbations subsequently plotted with MATLAB.

Figure 2 shows the initial condition of the optimal perturbation for spanwise wavenumber $k_{z}=2.5685$ (figure $2 a$ ) and $k_{z}=11.5574$ (figure $2 b$ ), and for various optimization times $T$. The wall-normal ( $v_{\text {opt }}$, black solid lines) and spanwise ( $w_{\text {opt }}$, dash-dotted lines) velocity components of the optimal perturbations do not evolve in time and are the same for any optimization time $T$. The thin grey lines show 
(a)

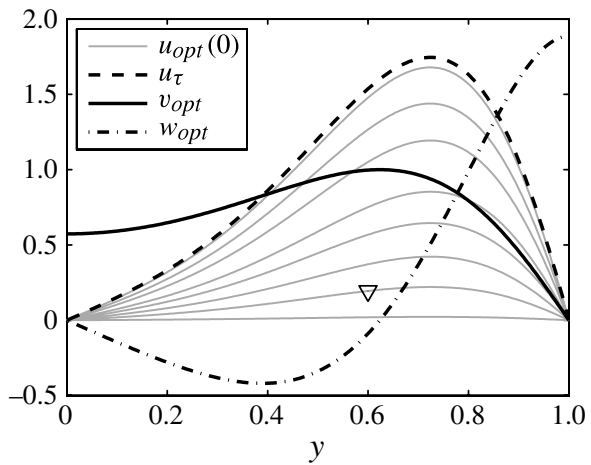

(b)

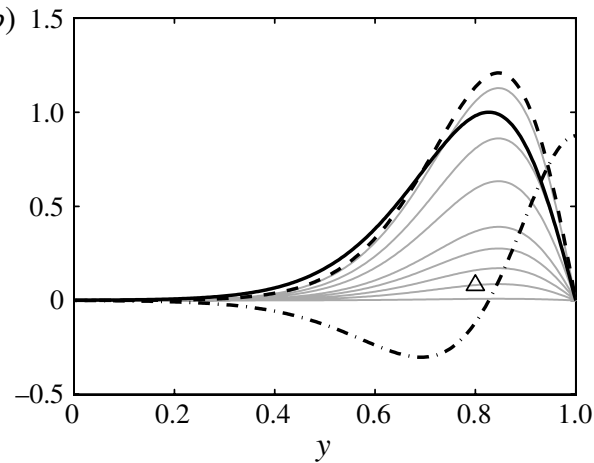

FIgURE 2. Components of the velocity of the optimal initial perturbation to Poiseuille flow for different $T$ and for $(a) k_{z}=2.5685$ and $(b) k_{z}=11.5574$. The different fields are normalized so that $\max \left(v_{\text {opt }}\right)=1$. Only half of the domain with $y \geqslant 0$ is shown, since all fields are antisymmetric about zero except for $v_{\text {opt }}$ which is symmetric. The thin grey lines correspond, from top to bottom, to the streamwise velocity $u$ of the optimal initial condition for $T=0.1,0.5,1,2,3,5,10$ and 100 . The symbols $\nabla(a)$ and $\Delta(b)$ indicate the initial condition of the optimal perturbation $u_{\text {opt }}(0)$ for $T=10$, whose corresponding optimal responses are shown in figure 3 below.

the initial streamwise velocity $u_{\text {opt }}(t=0)$ for different optimization times decreasing from $T=100$ to $T=0.1$ going from bottom to top. As the optimization time $T$ decreases to zero, $u_{\text {opt }}(t=0)$ approaches $u_{\tau} \equiv-S v_{\text {opt }} \tau_{\text {opt }}$ (black dashed line) which corresponds to the (initial and final) optimal streamwise velocity in the limit $T \rightarrow 0$. Accordingly, $u_{\tau}$ is the streamwise velocity corresponding to the maximum instantaneous growth rate $\sigma_{\max }=1 / 2 \tau_{\text {opt }}$. For any finite optimization time $T$, the streamwise component of the optimal perturbation evolves from the initial condition $u_{\text {opt }}(0)=-S v_{\text {opt }} \bar{t}_{\text {opt }}$ at $t=0$, passes through $u_{\tau}$ at the time $t=\tau_{\text {opt }}-\bar{t}_{\text {opt }} \in[0, T]$ of maximum instantaneous growth rate, and ends at $u_{\text {opt }}(T)=-S v_{\text {opt }}\left(\bar{t}_{\text {opt }}+T\right)$ at time $t=T$. For $T=10$, the optimal initial conditions for the two $k_{z}$ shown in figure 2 are marked by the symbols $\nabla(a)$ and $\Delta(b)$, and the corresponding optimal responses (the optimal perturbations at the final time $t=T$ ) are shown in figure 3 .

In the present linear inviscid case, all qualitative properties of the optimal perturbations described in figures 2 and 3 are independent of the optimization time $T$. Perturbations experience algebraic growth which goes unbounded as time increases, and so does the optimal gain $G_{o p t}$ as the optimization time $T$ goes to infinity. Equation (2.22) implies that as $T \rightarrow \infty$, the optimal gain is asymptotic to

$$
G_{o p t} \sim \frac{T^{2}}{\tau_{o p t}^{2}},
$$

meaning that

$$
\frac{1}{\tau_{\text {opt }}}=\lim _{T \rightarrow \infty} \sqrt{\frac{G_{\text {opt }}(T)}{T^{2}}}
$$

is the coefficient of algebraic growth of the inviscid optimal perturbation.

Figure 3 shows the optimal response for $k_{z}=2.5685(a)$ and $k_{z}=11.5574(b)$ for $T=10$ (indicated in figure 2 by $\nabla(a)$ and $\Delta(b)$ ) and makes apparent the periodic 

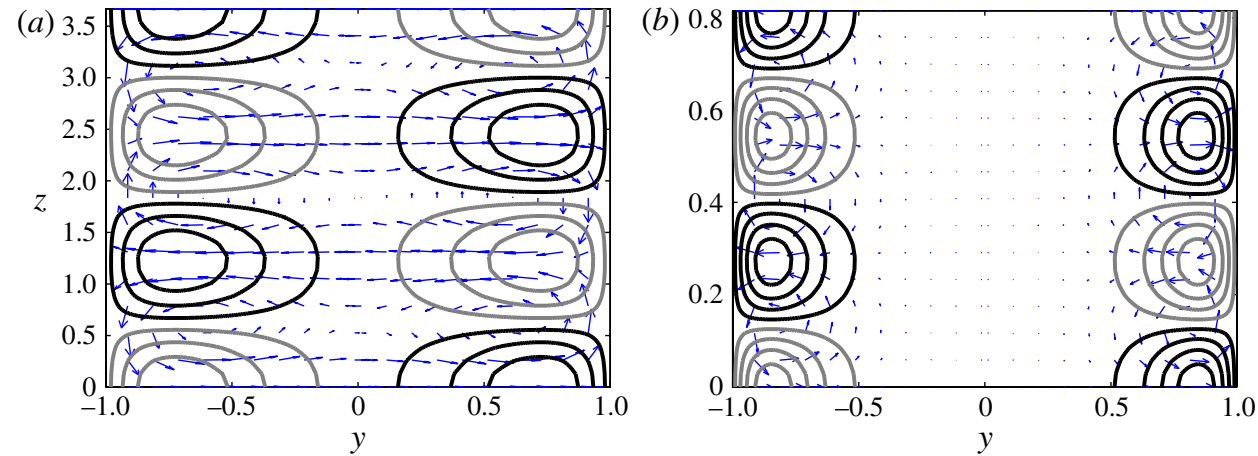

FIgURE 3. (Colour online) Spatial structure of the optimal perturbation to Poiseuille flow for $T=10$ and for $(a) k_{z}=2.5685$ and $(b) k_{z}=11.5574$. The arrows represent the $\left(v_{\text {opt }}, w_{\text {opt }}\right)$ field in the $(y, z)$ plane and the black (grey) lines show positive (negative) contour levels of $u_{\text {opt }}(t=T=10)$. The black (grey) contour lines are in intervals of 4(-4) starting from $\left|u_{\text {opt }}\right|=2$, the fields being normalized so that $\max \left(v_{\text {opt }}\right)=1$.

spanwise variation of $\boldsymbol{u}_{\text {opt }}$. The arrows represent the $\left(v_{\text {opt }}, w_{\text {opt }}\right)$ field in the $(y, z)$ plane, and the black (respectively grey) lines show positive (respectively negative) contour levels of $u_{\text {opt }}(t=T=10)$. Positive (respectively negative) contours are usually referred to as high (respectively low) speed streaks, and coincide with regions where the wallnormal velocity moves towards (respectively away from) the wall. This corresponds to the transport of base flow momentum by the perturbation, characteristic of the lift-up mechanism and similar to the optimal perturbations to Poiseuille flow computed previously by Butler \& Farrell (1992) in the viscous case. The comparison between $k_{z}=2.5685(a)$ and $k_{z}=11.5574(b)$ in figures 2 and 3 is straightforward: while the overall structure of both optimal perturbations is similar, their spatial localization is different and for the larger $k_{z}$ in figures $2(b)$ and $3(b), \boldsymbol{u}_{\text {opt }}$ is more concentrated close to the walls where the shear is maximum. The more localized perturbation is considerably more efficient in extracting energy from the base flow, with a coefficient of algebraic growth $\tau_{\text {opt }}^{-1}=1.384$ for the $k_{z}=11.5574$ case of figures $2(b)$ and $3(b)$, almost twice the $\tau_{\text {opt }}^{-1}=0.7787$ of the $k_{z}=2.5685$ case of figures $2(a)$ and $3(a)$.

Figure 4(a) shows the wall-normal velocity of the optimal perturbations $v_{\text {opt }}(y)$ for $y>0$ (since $v_{\text {opt }}(y)$ is symmetric) and different $k_{z}$. As $k_{z}$ increases, the maximum of $v_{\text {opt }}$ approaches the wall and, for $k_{z}$ sufficiently large, $v_{\text {opt }}(y)$ vanishes in the centre of the domain and gets localized at the wall. The dependence of the algebraic growth coefficient $\tau_{\text {opt }}^{-1}$ on $k_{z}$ is shown by the continuous line in figure $4(b)$, and the different symbols correspond to the $v_{\text {opt }}$ of figure $4(a)$. It can be observed that the algebraic growth or asymptotic gain coefficient $\tau_{o p t}^{-1}$ (and therefore the optimal gain for all $T$ ) increases monotonically with $k_{z}$.

Therefore, as the spanwise wavenumber $k_{z}$ increases, the inviscid optimal perturbations to Poiseuille flow become more efficient in extracting energy from the base flow as they become increasingly localized around the maximum shear. It seems from figure $4(a)$ that $v_{\text {opt }}$ adopts a self-similar limit shape as $k_{z}$ goes to infinity. At that point, the optimal perturbation should become insensitive to the details of the base flow profile, and the next section is devoted to deriving the asymptotics for this large $k_{z}$ regime. 

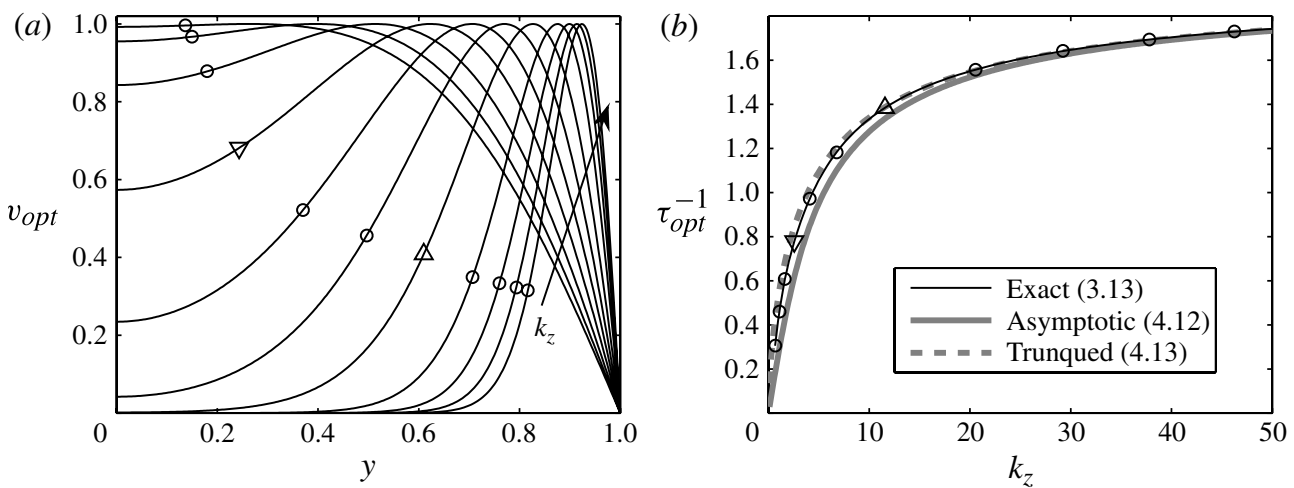

FIGURE 4. (a) Optimal wall-normal velocity $v_{\text {opt }}$ for Poiseuille flow and different spanwise wavenumber $k_{z}$. As hinted by the arrow, the optimal perturbations become increasingly localized close to the wall as $k_{z}$ increases; $v_{\text {opt }}$ is symmetric about zero and normalized so that $\max \left(v_{\text {opt }}\right)=1$. (b) Algebraic growth coefficient $\tau_{\text {opt }}^{-1}$ as a function of $k_{z}$. The black continuous line shows the result obtained from (3.13) for $n=1$. The $\nabla$ and $\Delta$ symbols in $(a, b)$ correspond to the fields shown in figures 2 and 3 . The remaining $(\bigcirc)$ symbols in $(b)$ indicate the $\left(k_{z}, \tau_{\text {opt }}^{-1}\right)$ values corresponding to the remaining $v_{\text {opt }}$ shown in $(a)$. The $k_{z}$ values of the curves in $(a)$ and symbols in $(b)$ are $k_{z}=0.65525,1.086,1.6433,2.5685,4.1116,6.7614,11.5574,20.5491,29.24,37.7881$ and 46.2499; which correspond respectively to $\alpha=0.1,0.25,0.5,1,2,4,8,16,24,32$ and 40 . The grey lines in $(b)$ show the asymptotic estimate of $\tau_{\text {opt }}^{-1}$ obtained from (4.12) (solid grey line) and from (4.13) evaluated up to order $\delta^{4 / 3}$ (dashed grey line). The latter asymptotic estimate is extremely precise even at small $k_{z}$, and the corresponding dashed line is barely visible behind the exact result shown by the solid black line.

\section{Large $k_{z}$ estimates of the inviscid optimal gain for free and bounded shear flow}

\subsection{Inflectional shear flow in an infinite domain}

Consider the case of an infinite domain where the shear is maximum at a $y$ location taken to be $y=0$. Then $S^{2}$ can be approximated around that maximum as

$$
S^{2}(y) \approx S_{o}^{2}+S_{o} S_{o}^{\prime \prime} y^{2}
$$

where $S_{o}^{2}$ and $S_{o}^{\prime \prime}$ are respectively the value of $S^{2}$ and the second derivative of $S$ at $y=0$, and $S_{o} S_{o}^{\prime \prime}<0$ so that $S_{o}^{2}$ is maximum. Substituting the approximation (4.1) into (2.19) yields

$$
-v^{\prime \prime}+\tau^{2} k_{z}^{2}\left|S_{o} S_{o}^{\prime \prime}\right| y^{2} v=k_{z}^{2}\left(\tau^{2} S_{o}^{2}-1\right) v
$$

Equation (4.2) is equivalent to the equation for the energy eigenstates of a quantum harmonic oscillator, and is treated in standard quantum mechanics textbooks (e.g. Ballentine 1998). In our variables, the quantization condition is

$$
\frac{k_{z}}{\sqrt{\left|S_{o} S_{o}^{\prime \prime}\right|}}\left(\tau S_{o}^{2}-\frac{1}{\tau}\right)=2 n-1,
$$


for $n=1,2, \ldots$ etc. Solving the quadratic equation (4.3) for $\tau>0$ yields

$$
\tau_{n}=\frac{\delta_{m}}{\left|S_{o}\right|}\left(n-\frac{1}{2}\right)+\frac{1}{\left|S_{o}\right|} \sqrt{\delta_{m}^{2}\left(n-\frac{1}{2}\right)^{2}+1},
$$

where $\delta_{m}=\left|k_{z}\right|^{-1} \sqrt{\left|S_{o}^{\prime \prime}\right| /\left|S_{o}\right|}$. For $n=1$, (4.4) gives an estimate of $\tau_{\text {opt }}=\tau_{1}$ for large $k_{z}$ (equivalently $\delta_{m} \ll 1$ ),

$$
\tau_{1}=\frac{\delta_{m}}{2\left|S_{o}\right|}+\frac{1}{\left|S_{o}\right|} \sqrt{\frac{\delta_{m}^{2}}{4}+1},
$$

which in turn yields an estimate for $G_{o p t}$ from (2.22).

The solutions of the approximate equation (4.2) are given by the corresponding set of eigenfunctions

$$
v_{n}=H_{n-1}\left(\gamma_{n} y\right) \exp \left(-\frac{\left(\gamma_{n} y\right)^{2}}{2}\right),
$$

where $\gamma_{n}=\sqrt{\tau_{n}\left|k_{z} S_{o} S_{o}^{\prime \prime}\right|}$ and $H_{n-1}$ is the Hermite polynomial of degree $n-1$ (Abramowitz \& Stegun 1964). For the leading mode $n=1$, the optimal perturbation $v_{\text {opt }}$ reduces to a Gaussian

$$
v_{o p t} \equiv v_{1}=\exp \left(-\frac{\left(\gamma_{1} y\right)^{2}}{2}\right)
$$

where

$$
\gamma_{1}=\left(\frac{\left|S_{o}^{\prime \prime}\right|}{2\left|S_{o}\right|}+\sqrt{\frac{S_{o}^{\prime \prime 2}}{4 S_{o}^{2}}+k_{z}^{2} \frac{\left|S_{o}^{\prime \prime}\right|}{\left|S_{o}\right|}}\right)^{1 / 2} .
$$

Expression (4.7) provides the asymptotic localization of the eigenfunction around the inflection point (maximum of $S^{2}$ ) as $k_{z} \rightarrow \infty$. Indeed, as $k_{z}$ goes to infinity, $\gamma_{1}$ in (4.7b) tends to $k_{z}^{1 / 2}\left(\left|S_{o}^{\prime \prime} / S_{o}\right|\right)^{1 / 4}$ and the optimal perturbation becomes

$$
v_{\text {opt }} \approx \exp \left(-\frac{1}{2} \sqrt{\frac{\left|S_{o}^{\prime \prime}\right|}{\left|S_{o}\right|}} k_{z} y^{2}\right),
$$

implying that the optimal perturbation becomes increasingly localized in a region whose width scales as $k_{z}^{-1 / 2}$.

We compare these asymptotic predictions with completely independent computations of the inviscid optimal perturbations for a tanh profile $U(y)=\tanh (y)$, which is commonly used to model free shear layers. For this, a pseudospectral code for the direct numerical simulation of the Navier-Stokes equations (NSE) linearized around a tanh profile has been used to compute the evolution of infinitesimal perturbations in the inviscid limit. The continuous adjoint NSEs have been implemented on the same code in order to retrieve the optimal perturbations through a power iteration algorithm that consists in solving the direct NSEs followed by the adjoint NSEs backwards in time (code and methodology as in Arratia et al. 2013).

The circles $(\bigcirc)$ in figure 5 show the optimal gain for $T=7$ as a function of $k_{z}$ for a $\tanh$ profile $U(y)=\tanh (y)$, as obtained by the direct-adjoint optimization algorithm. The dashed grey line shows the corresponding estimate given by (4.5) 


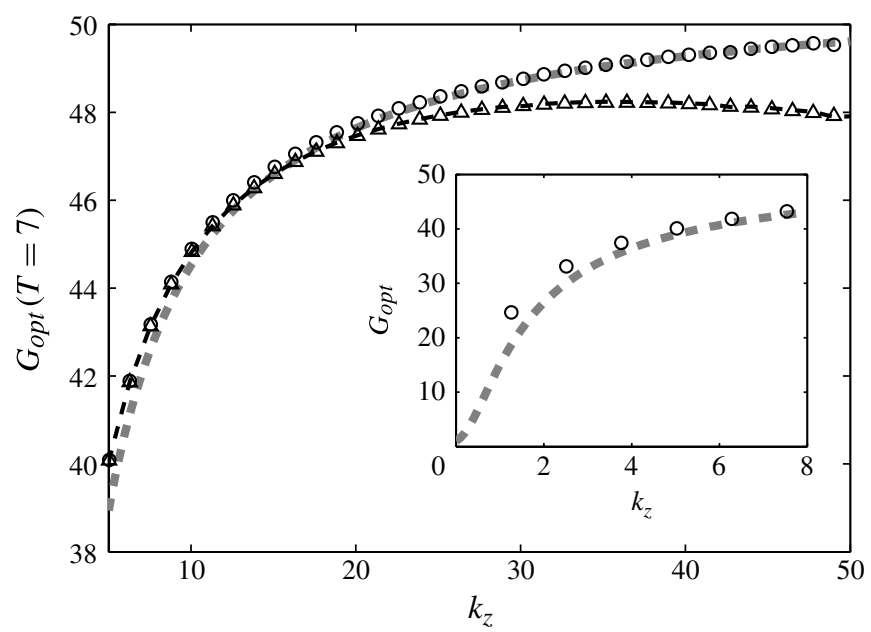

FIGURE 5. Optimal gain $G_{\text {opt }}$ as a function of $k_{z}$ for shear layer and $T=7$. The circles and triangles show $G_{o p t}$ for inviscid $(\bigcirc)$ and $R e=10^{6}(\triangle)$ perturbations to a tanh profile, numerically computed using a direct-adjoint technique implemented in a pseudospectral code (Arratia, Caulfield \& Chomaz 2013). The dashed grey line shows the estimate from (4.5) and the dashed black line shows the approximation of the viscous optimal gain obtained from (5.2) with the inviscid optimal gain for a tanh profile as $G_{i n v}$. The inset shows the inviscid data in the low $k_{z}$ region.

with $\delta_{m}=\sqrt{2} /\left|k_{z}\right|$ (the appropriate value for $U(y)=\tanh (y)$ ) substituted into (2.22). The estimate (4.5) yields the asymptotic behaviour of the optimal gain as $k_{z} \rightarrow \infty$, which is nevertheless apparently a good approximation even for small $k_{z}$.

The $v_{\text {opt }}(y)$ computed for the tanh profile are shown in figure $6(a)$ for $k_{z}=$ $2 \pi, 4 \pi, 8 \pi$ and $16 \pi$, as indicated in the figure. Remarkably, as in the Poiseuille flow case of $\S 3.3$, the optimal perturbations shown in figure $6(a)$ tend to become increasingly concentrated around the maximum shear (now at the inflection point $y=0)$ as $k_{z} \rightarrow \infty$. This is the limit at which (4.2) becomes relevant. Figure $6(b)$ shows the same numerically computed $v_{\text {opt }}$ as in figure $6(a)$, but normalized and plotted as a function of the rescaled coordinate $k_{z}^{1 / 2}\left(\left|S_{o}^{\prime \prime} / S_{o}\right|\right)^{1 / 4} y$. It can be seen that the asymptotic optimal perturbation given by (4.8) (grey line in figure $6 b$ ) constitutes a remarkably good approximation to the computed $v_{\text {opt }}$, becoming increasingly accurate as $k_{z}$ increases. These results support the appropriateness of the local approximation considered here.

In principle, the present, strictly local estimate may be turned into a full asymptotic solution by introducing an inner layer around the inflection point scaling as $k_{z}^{-1 / 2}$ where the solution is given by (4.6), matched with two surrounding outer layers where the solution is evanescent. This solution should be a good approximation around the inflection point even in a confined domain, provided the maximum is not at the wall.

\subsection{Bounded flow with maximum shear at a wall}

Consider now the domain $y \in[0, \infty)$ such that $S^{2}(y)$ has its maximum at $y=0$. Close to the boundary, $S^{2}$ can be approximated by

$$
S^{2}(y) \approx S_{o}^{2}+2 S_{o} S_{o}^{\prime} y,
$$



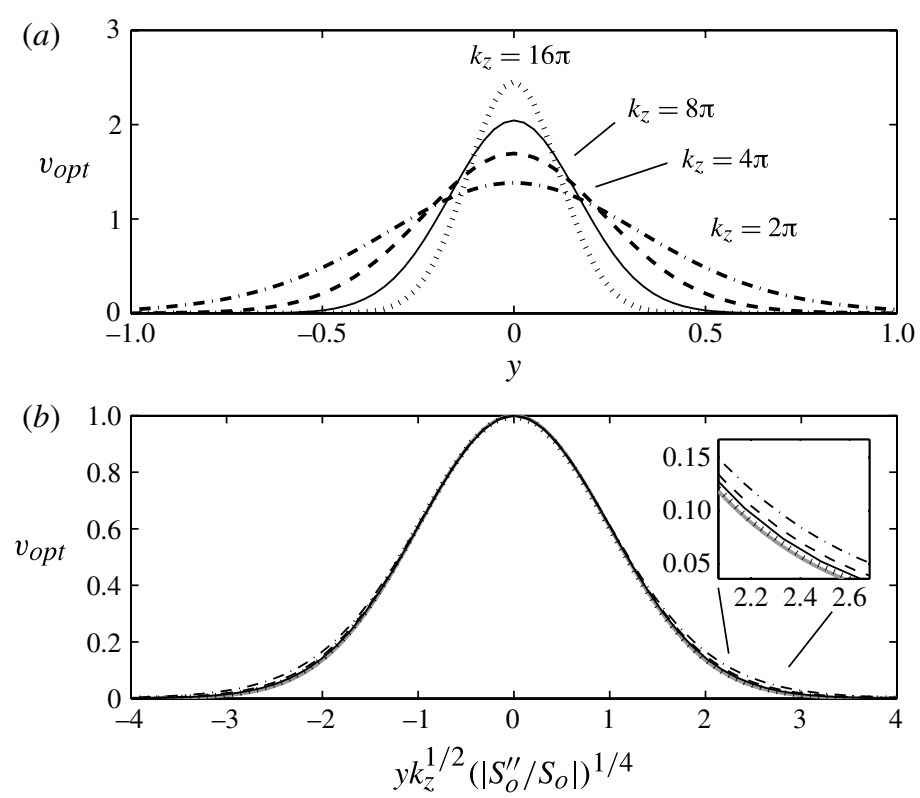

FIGURE 6. Optimal perturbation $v_{\text {opt }}$ numerically computed using a direct-adjoint technique implemented in a pseudospectral code (Arratia et al. 2013) for an inflectional $(\tanh (y))$ profile and $k_{z}=2 \pi$ (dash-dotted line), $k_{z}=4 \pi$ (dashed line), $k_{z}=8 \pi$ (continuous black line) and $k_{z}=16 \pi$ (dotted line). (a) $v_{\text {opt }}(y)$ showing the increasing localization of the optimal perturbations around the maximum of shear as $k_{z}$ increases. The different perturbations are normalized so that all optimal initial conditions (for $T=7$ as in figure 5) have the same energy. $(b)$ The same $v_{\text {opt }}$ as in $(a)$ but normalized (such that $v_{\text {opt }}(y=0)=1$ ) and plotted as a function of $y k_{z}^{1 / 2}\left(\left|S_{o}^{\prime \prime} / S_{o}\right|\right)^{1 / 4}$. The grey line shows the asymptotic eigenfunction $v_{\text {opt }}$ as defined in (4.8). The agreement with the direct computation is striking even for moderate $k_{z}\left(k_{z}=2 \pi\right)$.

where $S_{o} S_{o}^{\prime}<0$. Substituting (4.9) into (2.19) yields

$$
v^{\prime \prime}-\bar{Y} v=0
$$

where

$$
\bar{Y}=a^{1 / 3} y-b / a^{2 / 3}, \quad a=2 k_{z}^{2} \tau^{2}\left|S_{o} S_{o}^{\prime}\right| \quad \text { and } \quad b=k_{z}^{2}\left(\tau^{2} S_{o}^{2}-1\right), \quad(4.11 a, b, c)
$$

and the boundary conditions are $v=0$ at infinity and at the wall $\left(\bar{Y}=-b / a^{2 / 3}\right)$. Equation (4.10) can be solved in terms of Airy functions $\operatorname{Ai}(\bar{Y})$ (Abramowitz \& Stegun 1964). The Airy function $\operatorname{Ai}(\bar{Y})$ decays to zero when $\bar{Y}>0$ and is oscillatory for $\bar{Y}<0$. The boundary condition at the wall requires $-b / a^{2 / 3}$ to be a zero of $\operatorname{Ai}(\bar{Y})$ i.e.

$$
\left(\tau_{n}^{2} S_{o}^{2}-1\right)^{3}=-4 \alpha_{n}^{3} \delta^{2} \tau_{n}^{4} S_{o}^{4},
$$

where $\delta^{2}=S_{o}^{\prime 2} / S_{o}^{2} k_{z}^{2}$ and $\alpha_{n}<0$ is the $n$th zero of the Airy function. Condition (4.12) is a cubic equation for $\tau_{n}^{2}$ whose roots are known by standard formulae (Abramowitz \& Stegun 1964). It is, however, more instructive to consider a small $\delta$ expansion around the real solution $\tau_{1}^{2} S_{o}^{2}=1$ in the large wavenumber limit corresponding to $\delta^{2} \rightarrow 0$. The solution can be sought as a series expansion of the form $\tau_{1}^{2} S_{o}^{2}=1+\sum_{j \geqslant 1} c_{j} \delta^{2 j / 3}$ whose coefficients $c_{j}$ can be found order by order upon 


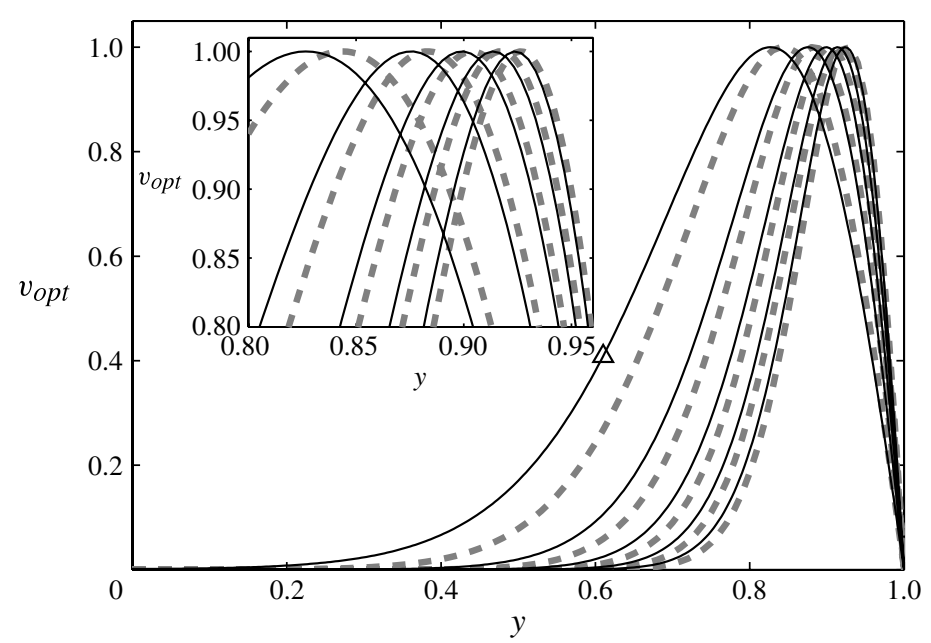

FIGURE 7. The continuous black lines show the $v_{\text {opt }}$ of figure $4(a)$ for $k_{z}=11.5574(\triangle)$ and larger. The dashed grey lines show the $v_{\text {opt }}$ approximation obtained from the Airy function solution of (4.10) with $\tau_{o p t}=\tau_{1}$ from (4.12) with $S_{o}=2, S_{o}^{\prime}=2$ and each of the corresponding $k_{z}$.

substitution into (4.12). Solving for the leading-order terms of this expansion, we obtain

$$
\tau_{1}^{2} S_{o}^{2}=1+\left(-4 \alpha_{1}^{3} \delta^{2}\right)^{1 / 3}+\frac{2}{3}\left(-4 \alpha_{1}^{3} \delta^{2}\right)^{2 / 3}+\frac{1}{3}\left(-4 \alpha_{1}^{3} \delta^{2}\right)+O\left(\delta^{8 / 3}\right) .
$$

The dashed line in figure $4(b)$ shows the estimate of the algebraic growth coefficient $\tau_{\text {opt }}^{-1}$ obtained from (4.13) up to order $\delta^{4 / 3}$ in the case of Poiseuille flow where the maximum shear is at the wall with $S_{o}=2$ and $S_{o}^{\prime}=2$. The agreement is excellent even when $k_{z}$ is small. It should be noted, however, that some error cancellation occurs for such good agreement, since the approximation obtained by directly solving (4.12) is slightly less accurate, as shown by the solid grey line in figure $4(b)$.

Now considering the localization of the perturbation near the wall, a comparison between the $v_{\text {opt }}$ predicted by the present asymptotic model and the exact $v_{\text {opt }}$ for Poiseuille flow is presented in figure 7. The continuous black lines show the $v_{\text {opt }}$ for Poiseuille flow and different $k_{z}$ already shown in figure 4(a). The dashed grey lines show $\operatorname{Ai}\left(\bar{Y}_{s}\right)$, the asymptotic optimal perturbation solution of (4.10) with $\bar{Y}_{s}=a^{1 / 3}(1-y)-b / a^{2 / 3}$ given from $(4.11)$ as $\bar{Y}$, but with $y$ replaced by $1-y$ to match the wall at 1 . The optimal parameter $\tau_{\text {opt }}=\tau_{1}$ is obtained by solving (4.12) numerically for each of the $k_{z}$. The asymptotic optimal wall-normal velocity $v_{\text {opt }}$ approaches the exact one as $k_{z}$ increases, especially close to the wall where the approximation (4.9) is valid. Thus, the local model given by (4.9)-(4.11) correctly describes the localization of the inviscid optimal perturbation next to the wall as $k_{z} \rightarrow \infty$. To determine how the localization of the perturbation scales with $k_{z}$, we substitute the leading-order solution of $\tau_{1}$ from (4.13) into the coordinate rescaling parameter $a$ defined in (4.11b), yielding $a=k_{z}^{2}\left|S_{o}^{\prime}\right| / 2\left|S_{o}\right|+O\left(k_{z}^{4 / 3}\right)$. As the rescaling of the coordinate in (4.11) is given by $a^{1 / 3}$, which at leading order when $k_{z} \rightarrow \infty$ becomes $a^{1 / 3} \sim\left(2\left|S_{o}^{\prime}\right| /\left|S_{o}\right|\right)^{1 / 3} k_{z}^{2 / 3}$, the optimal perturbations localize in an inner layer scaling as $k_{z}^{-2 / 3}$. As for the free shear flow, the present local estimate may be turned into a full asymptotic solution by introducing an inner layer where the wall-normal 


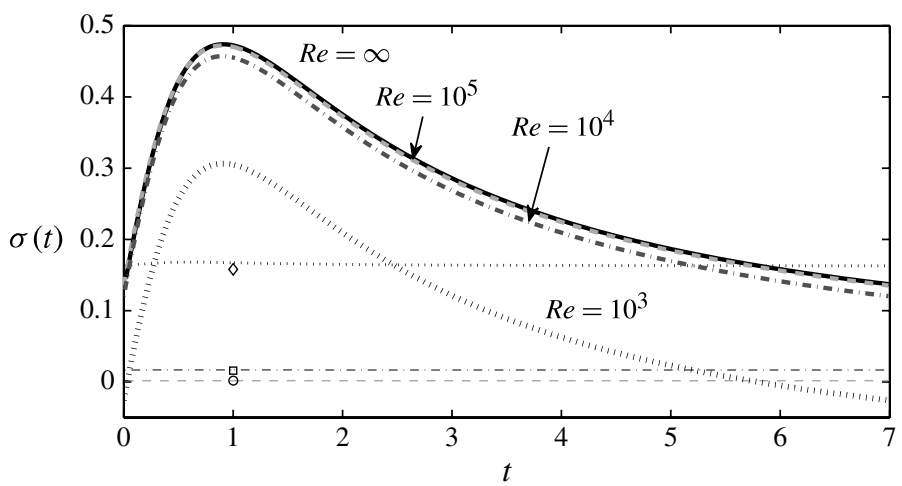

FIGURE 8. Instantaneous growth rate $\sigma(t)$ of the optimal perturbation to a tanh profile for $T=7$ and $k_{z}=4 \pi$, computed with the direct-adjoint method described in Arratia et al. (2013). Thick lines show $\sigma(t)$ in the inviscid (continuous black line), $R e=10^{5}$ (dashed light-grey line), $R e=10^{4}$ (dash-dotted dark-grey line), and $R e=10^{3}$ (dotted black line) cases. The difference between the viscous growth rates and the inviscid one, $\sigma_{i n v}-\sigma_{v}$, is shown by the corresponding thin lines: $R e=10^{5}$ (dashed light-grey line), $R e=10^{4}$ (dash-dotted dark-grey line), and $R e=10^{3}$ (dotted black line); the value of $R e^{-1} k_{z}^{2}$ corresponding to the same difference as predicted by (5.1) is shown by the symbols $\bigcirc\left(\operatorname{Re}=10^{5}\right), \square\left(\operatorname{Re}=10^{4}\right)$ and $\diamond\left(\operatorname{Re}=10^{3}\right)$.

coordinate is rescaled by $k_{z}^{-2 / 3}$, in which the inner layer solution is the Airy function vanishing at the wall and evanescent at infinity, as imposed by the matching condition with the outer layer.

\section{Viscous effects}

Except for condition (2.14), which only requires the stationarity of the base flow, the analysis presented in $\S 2$ is strictly valid only in the inviscid case. However, since the nature of the lift-up mechanism is inviscid, we expect some of the results to remain relevant for large Reynolds number in the viscous cases, for which the lift-up mechanism is known to lead to large but finite energy growth.

Figure 8 compares the instantaneous growth rate $\sigma(t)$ of the optimal perturbations to a tanh profile for an inviscid case (which we will denote by $\sigma_{i n v}(t)$ ) and for three viscous cases with different viscosities. In all cases $T=7$ and $k_{z}=4 \pi$, and the optimal perturbations are computed numerically with the direct-adjoint method described previously in $\S 4.1$. The section of trajectory realized by the inviscid optimal perturbation is such that $\sigma_{i n v}(0)=\sigma_{i n v}(T=7)$ according to condition (2.14). The condition (2.14) of equality between initial and final growth rate must also be satisfied in the viscous case, i.e. $\sigma_{v}(0)=\sigma_{v}(T)$ where $\sigma_{v}$ is the instantaneous growth rate of the viscous optimal perturbations. This is well verified by the $\sigma_{v}(t)$ obtained from the direct numerical computation of the viscous optimal perturbations for $R e=10^{5}, 10^{4}$ and $10^{3}$, where $R e$ is the Reynolds number given by $R e=v^{-1}$, with $v$ the kinematic viscosity in non-dimensionalization units (since $U(y)=\tanh (y)$ ). For $R e=10^{5}$ (dashed light-grey line), the growth rate $\sigma_{v}(t)$ is coincident with the inviscid one. For $R e=10^{4}$ (dash-dotted dark-grey line) the difference of $\sigma_{v}$ with $\sigma_{i n v}$ is visible but very small during the entire evolution of the two optimal perturbations, and for $R e=10^{3}$ (dotted black line) the difference is much larger and $\sigma_{v}$ becomes negative during the initial 
and final stage of the optimization interval. Despite the noticeable differences in growth rate for different $R e$, all the $\sigma_{v}(t)$ curves show a variation which is similar to the inviscid one: a sharp initial increase in $\sigma(t)$ leads to a maximum instantaneous growth rate just before $t=1$ that is followed by a slow decrease. The main difference between the cases is that the growth rate decreases as $R e$ decreases, consistent with the intuitive damping effect of viscosity on inviscid instability mechanisms.

For each of the $\sigma_{v}(t)$ shown by thick lines in figure 8 , thin lines (with the same line styles) show the difference between the inviscid and viscous optimal instantaneous growth rates $\sigma_{i n v}(t)-\sigma_{v}(t)$. The curves are almost perfectly horizontal lines for $R e=10^{5}$ (dashed light-grey line) and $R e=10^{4}$ (dash-dotted dark-grey line), and only a small vertical variation can be barely seen in the $R e=10^{3}$ (dotted black line) case. This indicates that the main effect of viscosity on the instantaneous growth rate of the optimal perturbations is to add an approximately constant dissipation rate.

One way to model the damping effect of viscosity is to approximate the viscous instantaneous growth rate $\sigma_{v}$ by $\sigma_{v i s}$ made from its inviscid counterpart $\sigma_{i n v}$ with a viscous correction

$$
\sigma_{v}(t) \sim \sigma_{v i s}(t) \equiv \sigma_{i n v}(t)-R e^{-1} k_{z}^{2}
$$

For the plane wave solutions considered in this study, approximation (5.1) takes into account exactly the effect of viscous diffusion in the $z$ direction, while neglecting it completely in the $y$ direction. The validity of this approximation implicitly relies on the assumption that at leading order for large $R e$, the shape of the optimal perturbation is that of the inviscid case. Using this shape assumption and the fact that the inviscid optimal perturbation is localized around the maximum of the shear with a thickness in the cross-stream direction scaling like $k^{-1 / 2}$ (for free shear layers), the viscous dissipation is indeed negligible in the $y$ direction and reduces to the equation (5.1). The value of the viscous dissipation rate $R e^{-1} k_{z}^{2}$ predicted by approximation (5.1) is marked by symbols in figure 8 for the different $R e$ considered. For $R e=10^{5}(\bigcirc)$ and $R e=10^{4}(\square)$, the marks lie on the essentially horizontal lines corresponding to the difference at each instant between the inviscid and the viscous instantaneous growth rate of the optimal perturbations, while for $\operatorname{Re}=10^{3}$ the mark $(\diamond)$ is slightly below. Thus, (5.1) provides a good approximation of the actual viscous growth rate in the large $R e$ limit.

Regarding the shape of the optimal perturbations, figure 9 shows a comparison of the initial and final cross-stream velocity of the numerically computed optimal perturbations of figure 8 . The different initial and final $v_{\text {opt }}$ in figure 9 have been normalized by the maximum of the corresponding initial cross-stream velocity $v_{\text {opt }}(0)$. For the final cross-stream velocities $v_{\text {opt }}(T=7)$, an additional multiplicative factor $\exp \left(R e^{-1} k_{z}^{2} T\right)$ has been included in order to compensate for the viscous diffusion as approximated in (5.1). The initial and final $v_{\text {opt }}$ for $R e=10^{5}$ and $R e=10^{4}$ shown in figure 9 are coincident with the inviscid result, and only for $R e=10^{3}$ do the diffusive effects become noticeable. The shape assumption is then legitimate for large $R e$, since the optimal cross-stream velocity $v_{\text {opt }}$ does not change at leading order with the Reynolds number.

When integrated over a time interval $T$, the viscous correction given by (5.1) provides an approximation $G_{v i s}(T)$ of the viscous gain $G_{v}(T)$ from its inviscid counterpart $G_{i n v}(T)$ given by

$$
G_{v}\left(T, k_{z}\right) \sim G_{v i s}\left(T, k_{z}\right) \equiv G_{i n v}\left(T, k_{z}\right) \exp \left(-2 R e^{-1} k_{z}^{2} T\right)
$$




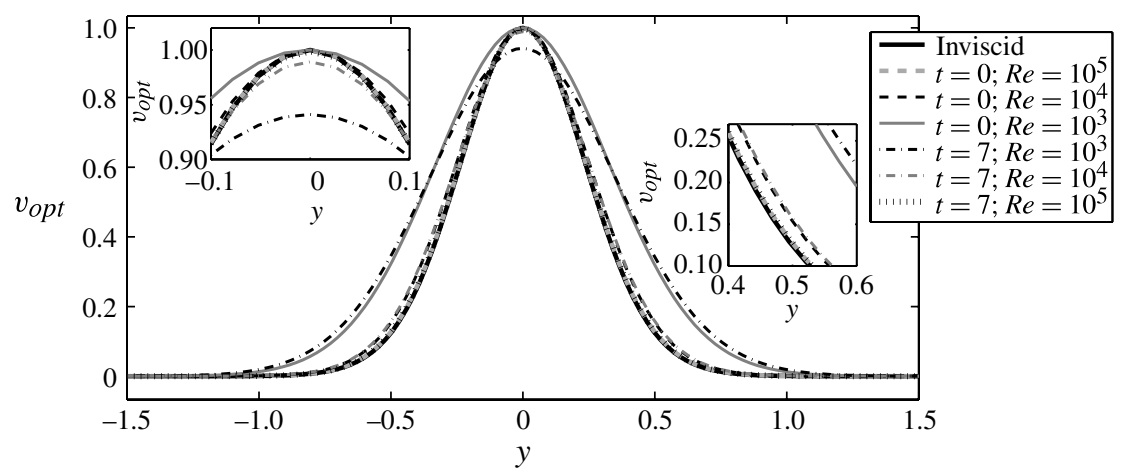

FIGURE 9. Initial and final cross-stream velocity $v_{\text {opt }}$ of the optimal perturbation to a tanh profile for $T=7$ and $k_{z}=4 \pi$, whose instantaneous growth rates are shown in figure 8 . The initial fields $v_{\text {opt }}(t=0)$ are normalized so that their maximum value is 1 . The final fields have been multiplied by the inverse of the viscous decay factor implied by (5.1), i.e. the lines labelled as $t=7$ for the different $R e$ correspond to $v_{\text {opt }}(T) \exp \left(R e^{-1} k_{z}^{2} T\right)$.

The viscous optimal gain as a function of $k_{z}$ for a tanh profile and $T=7$ is shown in figure 5 as numerically computed for $R e=10^{6}(\triangle)$ and as approximated from (5.2) (dashed black line) with $G_{i n v}$ given by the (numerically computed) inviscid optimal perturbation. For this large $R e$ and optimization time $T=7$, the agreement of (5.2) with the viscous direct computation is excellent in the whole range of $k_{z}$.

It is important to note that for the validity of the shape assumption implicit in (5.1) and (5.2), it is required that the optimization time $T$ be short compared to $T_{\nu}$, where $T_{\nu}$ is the time scale for diffusion in the $y$ direction. For large $k_{z}$ in the free shear layer case, this diffusive time scale will be dimensionally given by $T_{v} \sim\left|S_{o} / S_{o}^{\prime \prime}\right|^{1 / 2} / k_{z} v$ according to the asymptotic optimal perturbation solution of $\S 4.1$. Therefore, approximation (5.1) should be valid for non-dimensional optimization time $T \ll \operatorname{Re}_{z}^{-1}$ in the case of free shear layers.

For the case of wall-bounded shear flow, formulas (5.1) and (5.2) can be expected to be valid since the inviscid asymptotic solution predicts that the wall-normal (crossstream) extent of the optimal perturbation scales like $k_{z}^{-2 / 3}$, which means that the spanwise variation leads the dissipation in this case too. Assuming as in the previous case that the shape of the optimal perturbation is given by the inviscid solution, the asymptotic results of $\S 4.2$ imply that the time scale for diffusion in the wall-normal direction is now given by $T_{\nu} \sim\left|S_{o} / S_{o}^{\prime}\right|^{2 / 3} k_{z}^{-4 / 3} / \nu$, so that formulas (5.1) and (5.2) can now be valid for $T \ll \operatorname{Re} k_{z}^{-4 / 3}$. However, as we shall see, the shape assumption cannot be fully satisfied because of the boundary condition at the wall.

Figure 10(a) shows the optimal gain for Poiseuille flow and $T=0.1$ as a function of $k_{z}$. Results correspond to the exact solution for the inviscid case (solid black line), and to $R e=10^{5}(\bigcirc), \operatorname{Re}=10^{4}(\square)$ and $\operatorname{Re}=10^{3}(\diamond)$ for the viscous cases. The viscous optimal perturbations were numerically computed by directly solving the singular value decomposition of the linearized NSE operator obtained with a spectral collocation code (code provided by J. J. Soundar Jerome and used in Jerome et al. 2012: for details of the numerical procedure see their paper). For each of the different $R e$, the approximated viscous gain $G_{v i s}$ with the inviscid optimal gain as $G_{i n v}$ is shown by a dashed grey line. The approximation of the viscous optimal gain captures the $k_{z}$ dependence of the optimal perturbation and approaches the numerically 

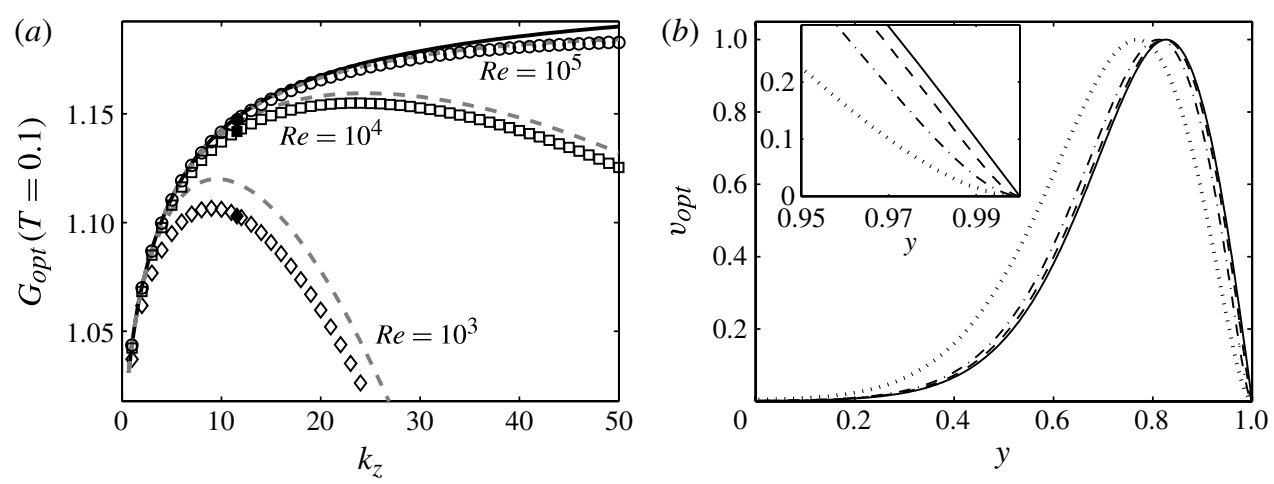

FIGURE 10. Viscous optimal perturbations for plane Poiseuille flow. (a) Optimal gain as a function of $k_{z}$ for $T=0.1$ (as in figure 2). The $\bigcirc, \square$ and $\diamond$ symbols correspond to the viscous case (numerically computed with the code used in Jerome, Chomaz \& Huerre 2012) at Reynolds numbers as specified in the figure. The solid symbols correspond to the $G_{\text {opt }}$ and $k_{z}$ of the $v_{o p t}$ shown in $(b)$. The continuous black line shows the inviscid result obtained from the exact solution (2.22) and (3.11). The dashed grey lines correspond, for each $R e$, to the approximation of the viscous optimal gain obtained from (5.2) with the inviscid optimal gain as $G_{i n v}$. (b) $v_{\text {opt }}(t=0)$ for $k_{z}=11.5574$ (highlighted with the solid symbols in $a$ ) for $R e=10^{3}$ (dotted line), $R e=10^{4}$ (dash-dotted line), $R e=10^{5}$ (dashed line) and the inviscid case (continuous line). The inset shows the different $v_{\text {opt }}$ close to the wall.

computed values as $R e$ increases. Thus, the inviscid optimal perturbations are relevant to the viscous case for Poiseuille flow as well.

Still, given the short optimization time $T=0.1$ considered, the differences between the approximated and the numerically computed viscous results in figure $10(a)$ are important, especially for $R e=10^{3}$. This is not surprising since the viscous optimal perturbation must satisfy no-slip boundary conditions in the presence of a wall. Figure $10(b)$ shows $v_{o p t}$ at $t=0$ for the viscous optimal perturbations with $k_{z}=11.5574$ (marked with solid symbols in figure 10a) and the corresponding inviscid optimal perturbation (also shown in figure $3 b$ and marked with a $\Delta$ in figures $4(a)$ and 7). For $\operatorname{Re}=10^{3}$ (dotted line), the shape of $v_{\text {opt }}$ is similar to the inviscid one (continuous line) but slightly displaced from the wall situated at $y=1$. For $R e=10^{4}$ and $R e=10^{5}$ (dash-dotted and dashed line respectively), the $v_{\text {opt }}$ are still a little displaced but very close to their inviscid counterpart, getting closer as $R e$ gets larger. The inset shows a closer look at the shape of the different $v_{\text {opt }}$ near the wall. While the inviscid $v_{\text {opt }}$ starts with a constant slope from the wall, the curves of the viscous $v_{\text {opt }}$ start with a zero slope from the wall and rapidly turn to become parallel to the inviscid curve after a small distance from the wall. Thus, the difference originates at the wall where the viscous streamwise velocity must also satisfy $v_{o p t}^{\prime}=0$ as the boundary condition, which is required to enforce the no-slip boundary condition on $w_{\text {opt }}$.

We may also note that the streamwise velocity of the inviscid optimal perturbation $u_{\text {opt }}$ satisfies the no-slip boundary condition, so no extra boundary conditions are required on the inviscid optimal perturbations besides $v_{o p t}^{\prime}=0$. Therefore, in order to uniformly approximate the viscous optimal perturbation in the presence of a wall, one may start from the inviscid $v_{\text {opt }}$ plus a viscous boundary layer next to the wall in order to impose the extra condition $v_{o p t}^{\prime}=0$. Using the viscosity $v$, the maximum shear $S_{o}$ and the length scale of the inviscid optimal perturbation near the wall $a^{-1 / 3}$ (see 
(4.11)), the width $\delta_{v}$ of the boundary layer of the viscous optimal perturbation can be estimated as $\delta_{v} \sim v k_{z}^{2 / 3}\left|S_{o}^{\prime}\right|^{1 / 3}\left|S_{o}\right|^{-4 / 3}$. Once a viscous approximation of the crossstream velocity has been determined, the remaining components of the approximated optimal perturbation will follow from $v_{\text {opt }}$ as in the inviscid case. The construction of such an approximation goes beyond the scope of the present paper.

\section{Conclusion}

We have formally solved the optimal perturbation problem for longitudinal (streamwise independent) perturbations to arbitrary inviscid parallel flow at arbitrary optimization time. The key to this derivation was the use of the time invariance of the governing equations. For every problem invariant under time translation, the optimization over all initial conditions can be decomposed into an optimization of the time phase along trajectories and an optimization on a transverse codimension-1 subspace $\left(\mathscr{S}_{\perp}\right)$ crossed by all trajectories. Here, $\mathscr{S}_{\perp}$ is chosen as the plane wherein $u$ and $S v_{o}$ are orthogonal in the Euclidean inner product. Then, optimizing first along trajectories imposes (2.14), requiring that the instantaneous energy growth rate must be equal at the initial and final times $\sigma(0)=\sigma(T)$. This property is generic to any linear optimization problem requiring only the stationarity of the base flow. It is also valid for nonlinear equations if no extra conditions such as a prescribed magnitude for the initial energy are imposed.

For the simple case of longitudinal perturbations to parallel shear flow considered here, the equality of initial and final growth rates for a given $T$ can be explicitly determined on each trajectory. We have then shown that, for each trajectory, the maximum amplification for any optimization time $T$ depends on a single real parameter $\tau$ which corresponds to the inverse of the slope of the algebraic growth, and which must be minimized to find the optimal perturbation. The optimization is then first solved for $\bar{u}_{o}$ giving $\bar{u}_{o}=0$, and then for $v_{o}$. Remarkably in this case, the optimal perturbations for any optimization time $T$ belong to the same trajectory in phase space. We have provided the expression of the optimal gain $G_{o p t}(T)$ as a function of $\tau$, which appears as the eigenvalue in an eigenvalue problem which is independent of $T$ and also yields the shape of the optimal perturbation and a set of sub-optimal perturbations.

We have solved this eigenvalue problem to provide exact analytical solutions for the inviscid longitudinal optimal perturbations for plane Couette flow, a shear layer profile of the form $2 U_{o} \arctan (\tanh (y / 2 L))$, and plane Poiseuille flow. For Poiseuille flow, the prediction of this novel inviscid solution compares well with the optimal gain and perturbations computed in the viscous case for large Reynolds number by solving numerically the singular value decomposition (Jerome et al. 2012). Asymptotic approximations in the limit $k_{z} \rightarrow \infty$ have been determined in two generic cases: inflectional shear and wall-bounded shear with the maximum at the wall. For inflectional shear (with a maximum within the domain), the $y$-extension of the optimal perturbation scales as $k_{z}^{-1 / 2}$, becoming increasingly localized at the inflection point as $k_{z}$ increases. For maximum shear at a wall, the localization of the optimal perturbation at the wall is stronger, with the size along the wall-normal direction scaling as $k_{z}^{-2 / 3}$. In both cases, asymptotic estimates of the optimal gain are given as a function of the maximum shear and its first (respectively second) derivative at the wall (respectively at the inflection point). Both asymptotic predictions have been tested: in the free shear layer case by comparing to the directly computed optimal gain and optimal perturbations to a tanh profile using the direct-adjoint method (Arratia et al. 2013), 
and by comparing to the optimal perturbations and to the slope of the algebraic growth rate for Poiseuille flow, derived in the present paper, in the case of wall-bounded shear flow.

We have also studied the relevance of our results to the viscous case and shown that the viscous optimal perturbations approach the inviscid ones as $R e$ increases. The main effect of viscous damping is strongly dependent on $k_{z}$, and most of this dependence on the optimal gain can be captured with a simple correction of the inviscid result, justified by the asymptotic shape of the optimal perturbations and in particular the estimate of the typical variation scale in $y\left(k_{z}^{-1 / 2}\right.$ for free shear and $k_{z}^{-2 / 3}$ for wallbounded flow). The effect of viscosity on the optimal perturbation remains important at a wall, an effect that might be included in the inviscid optimal perturbation by adding a viscous boundary layer in order to impose that $v_{o p t}^{\prime}=0$ as the boundary condition.

\section{Acknowledgements}

We are very grateful to J. S. Jerome for providing the code to compute the viscous optimal perturbations for Poiseuille flow, and to C. P. Caulfield and F. Gallaire for carefully reading the manuscript and giving useful comments. We thank the insightful remarks and constructive criticisms from three anonymous referees. C.A. acknowledges financial support for his $\mathrm{PhD}$ from the Chilean and French governments.

\section{Appendix A. Orthogonal decomposition of the evolution of perturbations}

In this appendix we review some mathematical properties of the optimal perturbation equation (2.19) and describe how the evolution of general perturbations can be decomposed in its eigenfunctions. If the domain is finite in $y$ and $S(y)$ is nowhere zero, it can be shown rigorously (Teschl 2012, Theorem 5.11) that there are infinitely countable real eigenvalues $\tau_{n}$, the corresponding eigenfunctions $v_{n}$ can be chosen real and form a complete orthonormal set under the inner product $\left\{v_{m}, v_{n}\right\} \equiv\left\langle v_{m} S, v_{n} S\right\rangle$, i.e. $\left\{v_{m}, v_{n}\right\} \propto \delta_{m n}$ where $\delta_{m n}$ is the Kronecker delta. The eigenvalues $\tau_{n}$ can be ordered in ascending order $\tau_{1}, \tau_{2}, \ldots$ where $\tau_{\text {opt }}=\tau_{1}$, each $\tau_{n}$ corresponding to an eigenfunction $v_{n}$ possessing $n-1$ nodes (Teschl 2012, Theorem 5.17). Note from (2.1a) that the set of eigenfunctions $\left\{v_{n}\right\}$ with the inner product $\{\cdot, \cdot\}$ (or equivalently $\left\{S v_{n}\right\}$ with the inner product $\langle\cdot, \cdot\rangle$ ) provides an orthogonal decomposition for the forcing of the streamwise velocity $u$ by the crossstream velocity $v$. Similarly, for any initial condition, the streamwise velocity $u(y, t)$ can be decomposed using the $v_{n}$ into

$$
u(y, t)=\bar{u}_{o}(y)-\left(t+\bar{t}_{o}\right) \sum_{n \geqslant 1} c_{n} v_{n}(y) S(y),
$$

where the sum coefficients are complex and given by $c_{n}=\left\{v_{n}, v_{o}\right\}$ (with $\left\{v_{n}, v_{n}\right\}=$ $\left\|S v_{n}\right\|^{2}=1$ as normalization), and are determined solely by $v_{o}$, while $\bar{t}_{o}$ and $\bar{u}_{o}$ depend also on $u_{o}$ and are given respectively by (2.6) and (2.9). Although $\bar{u}_{o}$ is in general not orthogonal to the $v_{n}(y) S(y)$ terms of the sum in (A 1) independently, it is by construction orthogonal to the full sum in the two-dimensional Euclidean norm $\operatorname{Re}(\langle\cdot, \cdot\rangle)$ which provides the energy contribution of the crossed terms (see (2.4) and the discussion after (2.9)), and using the orthogonality of the $v_{n}$ we obtain that (twice) 

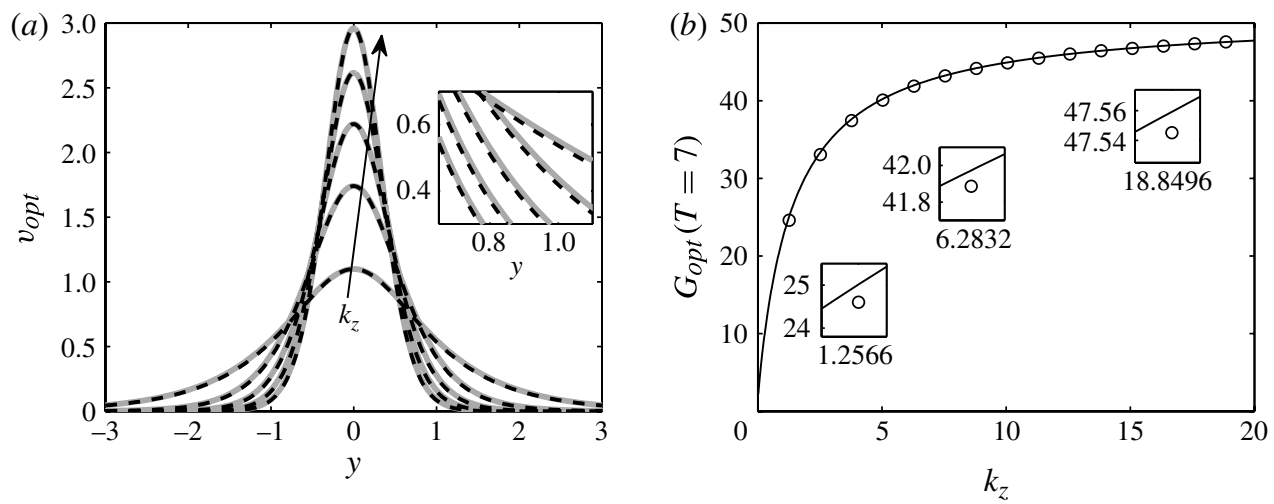

FIGURE 11. (a) Optimal cross-stream velocity $v_{\text {opt }}$ for the $\operatorname{arcttanh}$ profile $U(y)=$ $\arctan (\tanh y / \sqrt{2}) / \sqrt{2}$ (solid grey lines) and for the tanh profile $U(y)=\tanh (y)$ (dashed black lines) for different spanwise wavenumbers $k_{z}$. The values of the spanwise wavenumber $k_{z}$ are $k_{z}=0.4 \pi, 0.8 \pi, 1.2 \pi, 1.6 \pi$ and $2 \pi$, increasing as the maximum value of $v_{\text {opt }}$ increases, as hinted by the arrow. The different $v_{\text {opt }}$ for the tanh profile are normalized so that their integral $\int v_{\text {opt }} \mathrm{d} y$ is the same for the different $k_{z}$. For each $k_{z}$, the $v_{\text {opt }}$ for the arcttanh profile are normalized by the maximum value of the tanh profile $v_{\text {opt }}$ for the corresponding $k_{z}$. (b) Optimal gain for the arcttanh (solid black line) and for the tanh profile (O) for $T=7$ as a function of $k_{z}$. The insets show close-ups for $k_{z}=0.4 \pi, 2 \pi$ and $6 \pi$.

the streamwise kinetic energy of the perturbation is

$$
\|u(t)\|^{2}=\left\|\bar{u}_{o}\right\|^{2}+\left(t+\bar{t}_{o}\right)^{2} \sum_{n \geqslant 1}\left|c_{n}\right|^{2},
$$

and the total energy of the perturbation is

$$
E(t)=\bar{E}_{o}+\frac{\left(t+\bar{t}_{o}\right)^{2}}{2} \sum_{n \geqslant 1}\left|c_{n}\right|^{2} .
$$

Equations (A 1) and (A 3) show that the set of eigenfunctions of (2.19) provides a complete orthogonal basis to describe the time-evolving part of an arbitrary perturbation.

\section{Appendix B. Comparison of the optimal perturbations for the arcttanh and tanh profiles}

A comparison between exact optimal perturbations to the arcttanh profile $U(y)=$ $2 U_{o} \arctan (\tanh y / 2 L)$ with $U_{o}=L=1 / \sqrt{2}$, and numerically computed (as described in $\S 4.1)$ optimal perturbations to the $\tanh$ profile $U(y)=\tanh (y)$, is shown in figure 11. The values $U_{o}=L=1 / \sqrt{2}$ are obtained by imposing that the maximum shear $S_{o}$ and its second derivative $S^{\prime \prime}$ at $y=0$ (at the maximum) are equal for both profiles, a requirement that follows from the analysis of $\S 4.1$. Figure 11 $(a)$ shows $v_{\text {opt }}(y)$ from solution $(3.10 b)$ to the arcttanh profile (solid grey lines) and for the tanh profile (dashed black lines) for five different values of $k_{z}$ between $0.4 \pi$ and $2 \pi$, increasing as the maximum of $v_{\text {opt }}$ increases. For each of the spanwise wavenumbers $k_{z}$ considered, the $v_{\text {opt }}$ curves coincide almost perfectly even for the smallest $k_{z}$ considered, the difference being barely visible in the inset. Figure 11(b) shows the 
corresponding optimal gain as a function of $k_{z}$ for both profiles and for $T=7$. The agreement between the optimal gains is also very good and the values become closer as $k_{z}$ increases, as can be seen from the different vertical scales of the insets. Thus, the exact solution (3.10) for the $\operatorname{arcttanh}$ profile $U=\arctan (\tanh y / \sqrt{2}) / \sqrt{2}$, provides an excellent approximation for $v_{\text {opt }}$ and the optimal gain for the $\tanh$ profile $U=\tanh (y)$. This may seem surprising if one considers that when $U_{o}=L=1 / \sqrt{2}$, the velocity jump for the arcttanh profile is more than $10 \%$ larger than the velocity jump for the tanh profile.

\section{REFERENCES}

Abramowitz, M. \& Stegun, I. 1964 Handbook of Mathematical Functions. Dover.

Arratia, C., Caulfield, C. P. \& Chomaz, J.-M. 2013 Transient perturbation growth in time-dependent mixing layers. J. Fluid Mech. 717, 90-133.

Ballentine, L. E. 1998 Quantum Mechanics. Prentice Hall.

Butler, K. M. \& FARREll, B. F. 1992 Three-dimensional optimal perturbations in viscous shear flow. Phys. Fluids A 4 (8), 1637-1650.

Ellingsen, T. \& Palm, E. 1975 Stability of linear flow. Phys. Fluids 18 (4), 487-488.

FARrell, B. F. \& IOANNOU, P. J. 1993 Optimal excitation of three-dimensional perturbations in viscous constant shear flow. Phys. Fluids A 5 (6), 1390-1400.

Gustavsson, L. H. 1991 Energy growth of three-dimensional disturbances in plane Poiseuille flow. J. Fluid Mech. 224, 241-260.

Hanifi, A. \& Henningson, D. S. 1998 The compressible inviscid algebraic instability for streamwise independent disturbances. Phys. Fluids 10 (8), 1784-1786.

Jerome, J. J. S., Chomaz, J.-M. \& Huerre, P. 2012 Transient growth in Rayleigh-BénardPoiseuille/Couette convection. Phys. Fluids 24 (4)044103.

LANDAHL, M. T. 1980 A note on an algebraic instability of inviscid parallel shear flows. J. Fluid Mech. 98 (2), 243-251.

Landau, L. D. \& Lifshitz, E. M. 1977 Quantum Mechanics: Non-Relativistic Theory. Pergamon.

Malik, M., Alam, M. \& DeY, J. 2006 Nonmodal energy growth and optimal perturbations in compressible plane Couette flow. Phys. Fluids 18 (3)034103.

Moffatt, H. K. 1967 The interaction of turbulence with strong wind shear. In Atmospheric Turbulence and Radio Wave Propagation (ed. A. M. Yaglom \& V. I. Tatarski), pp. 139-154.

Reddy, S. C. \& Henningson, D. S. 1993 Energy growth in viscous channel flows. J. Fluid Mech. 252, 209-238.

Schmid, P. J. \& Henningson, D. S. 2001 Stability and Transition in Shear Flows. Springer.

Teschl, G. 2012 Ordinary Differential Equations and Dynamical Systems. Graduate Studies in Mathematics Series, vol. 140, American Mathematical Society. 